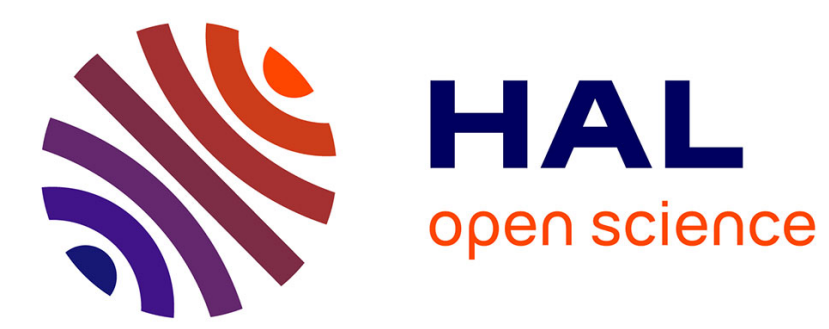

\title{
On noise generation in low Reynolds number temporal round jets at a Mach number of 0.9
}

Christophe Bogey

\section{To cite this version:}

Christophe Bogey. On noise generation in low Reynolds number temporal round jets at a Mach number of 0.9. Journal of Fluid Mechanics, 2019, 859, pp.1022 - 1056. 10.1017/jfm.2018.864 . hal-02333794

\section{HAL Id: hal-02333794 \\ https://hal.science/hal-02333794}

Submitted on 24 Nov 2020

HAL is a multi-disciplinary open access archive for the deposit and dissemination of scientific research documents, whether they are published or not. The documents may come from teaching and research institutions in France or abroad, or from public or private research centers.
L'archive ouverte pluridisciplinaire HAL, est destinée au dépôt et à la diffusion de documents scientifiques de niveau recherche, publiés ou non, émanant des établissements d'enseignement et de recherche français ou étrangers, des laboratoires publics ou privés. 


\title{
On noise generation in low Reynolds number temporal round jets at a Mach number of 0.9
}

\author{
Christophe Bogey ${ }^{1} \dagger$ \\ ${ }^{1}$ Laboratoire de Mécanique des Fluides et d'Acoustique, UMR CNRS 5509, \\ Ecole Centrale de Lyon, Université de Lyon, 69134 Ecully Cedex, France
}

(Received ${ }^{* * *}$ )

Two temporally-developing isothermal round jets at a Mach number of 0.9 and the Reynolds numbers of 3,125 and 12,500 are simulated in order to investigate noise generation in high-subsonic jet flows. Snapshots and statistical properties of the flow and sound fields, including mean, rms and skewness values, spectra and auto- and crosscorrelations of velocity and pressure, are presented. The jet at a Reynolds number of 12,500 develops more rapidly, exhibits more fine turbulent scales, and generates more high-frequency acoustic waves than the other. In both cases, however, when the jet potential core closes, mixing-layer turbulent structures intermittently intrude on the jet axis, and strong low-frequency acoustic waves are emitted in the downstream direction. These waves are dominated by the axisymmetric mode and are significantly correlated with centerline flow fluctuations. These results are similar to those obtained at the end of the potential core of spatially-developing jets. They suggest that the mechanism responsible for the downstream noise component of these jets also occurs in temporal jets, regardless of the Reynolds number. This mechanism is revealed by averaging the flow and pressure fields of the present jets using a sample synchronization with the minimum values of centerline velocity at potential-core closing. A spot characterized by a lower velocity and a higher vorticity level relative to the background flow field is found to develop in the interfacial region between the mixing layer and the potential core, to strengthen rapidly and reach a peak intensity when arriving on the jet axis, and then to break down. This is accompanied by the growth and decay of a hydrodynamic pressure wave, propagating at a velocity which, initially, is close to 65 per cent of the jet velocity and slightly increases, but quickly decreases after the collapse of the high-vorticity spot in the flow. During that process, sound waves are radiated in the downstream direction.

\section{Introduction}

After more than sixty years of research, the characteristics of the acoustic sources are well established for supersonic jets, but this is not the case for subsonic jets due to limited knowledge of jet flow turbulence, as pointed out in the reviews by Tam (1995, 1998). In particular, despite the development of comprehensive theories on the generation of aerodynamic noise (Lighthill 1952; Crighton 1975), the nature of the sources has been a matter of debate for a long time. Using localization techniques as in Chu \& Kaplan (1976) and Fisher et al. (1977) for instance, the source distribution along the axial direction in subsonic jets was, however, shown to depend on the Strouhal number $\operatorname{St}_{D}=f D / u_{j}$, where $f$ is the frequency, and $D$ and $u_{j}$ are the jet exit diameter and velocity. Overall, high-frequency sound sources are located near the nozzle exit, whereas low-frequency sources lie in the vicinity of the end of the jet potential core. The sound spectra measured

$\dagger$ Email address for correspondence: christophe.bogey@ec-lyon.fr 
in the acoustic field were also found to be dominated by low-frequency components in the downstream direction, but to be broadband in the sideline and upstream directions, see the far-field measurements of Mollo-Christensen et al. (1964) for example. These observations, and other experimental and numerical findings reported in Tam (1998), Panda et al. (2005) and Tam et al. (2008), and in Bogey et al. (2003) and Bogey \& Bailly (2006, 2007), among others, suggested the presence of two noise components, namely a low-frequency component dominating in the downstream direction, marked by intermittent aspects (Juvé et al. 1980), and a broadband component with a relatively uniform directivity. Thanks notably to the semi-empirical model of Tam \& Auriault (1999), the broadband component was identified as the noise of the fine-scale turbulence of the jet flow. The low-frequency component, emerging typically around $\mathrm{St}_{D}=0.2$, was attributed to the flow large-scale structures and/or instability waves. Unfortunately, despite the efforts of many researchers, its generation mechanism is still not clearly understood.

Some properties of the source radiating the downstream jet noise component have been inferred from experimental and numerical data. It appears to be located in the vicinity of the end of the potential core, where the mixing layers merge. This contention is supported by the source distributions obtained using localization techniques (Chu \& Kaplan 1976; Fisher et al. 1977), but also by cross-correlations calculated between flow quantities inside the jets and sound pressure outside (Seiner 1974; Schaffar 1979; Panda et al. 2005; Panda 2007; Bogey \& Bailly 2007; Grizzi \& Camussi 2012) Indeed, significant values of correlations are found between the flow on the jet axis near the end of the potential core and the acoustic waves emitted at small angles relative the downstream direction. The corresponding noise generation mechanism seems also to be related to the fact that at the end of the potential core, shear-layer turbulent structures intrude intermittently and quasi-periodically into the jet (Bogey et al. 2003). This bursting-like event was described in Tinney et al. (2008) as a volcano-like eruption forcing a highly coherent mass of fluid through the centre of the jet. It was moreover noted to occur for only a small fraction of the time but for a very high level of turbulent energy, with a regularity matching with the jet's peak Strouhal number.

For a long time (Mollo-Christensen 1967), jet noise sources have also been associated with the streamwise evolution of instability waves, which can be used to describe the dynamics of the flow coherent structures. The modelling of such waves in theoretical studies, e.g. by Kempton \& Ffowcs Williams (1978) and Tam \& Morris (1980), just to mention a few well-known pioneers in that field, provided features consistent with jet far-field experimental data. In Crighton \& Huerre (1990), in particular, it offered an explanation for the superdirective character of jet noise. Due to the spreading of the jet flow, the instability waves grow, saturate and decay, which results in a small fraction of flow components with supersonic phase velocity, radiating noise downstream, as illustrated in Tam et al. (2008), Suzuki (2010) and Jordan \& Colonius (2013). Nevertheless, this phenomenon alone appears to underestimate the actual sound pressure levels of jets, indicating that other important characteristics observed during the collapse of coherent structures at the end of the potential core, such as intermittency and nonlinearity, must be included in the model (Cavalieri et al. 2011; Suzuki 2013) to increase the radiated noise levels. For that purpose, Kastner et al. (2006) notably proposed to use a truncated wave packet instead of a symmetric wave packet to mimic the rapid breakdown of the flow large-scale structures in the model.

In order to isolate the source of the downstream jet noise component, it can be worth dealing with a reduced or simplified jet flow configuration. One possibility is to consider temporally-developing flows, as in the present work. The amplification of 
temporally-growing disturbances in an hyperbolic-tangent velocity profile was investigated by Michalke (1964) using linear stability theory. Temporally-developing mixing layers have been calculated in several studies, including those by Comte et al. (1992), Vreman et al. (1996) and Freund et al. $(2000 a, b)$, in order to describe the turbulent development and the compressibility effects in free shear flows. Simulations of temporal mixing layers have also allowed for studies of noise generation and propagation in such flows, refer to Fortuné et al. (2004) and Kleimann \& Freund (2008) for subsonic mixing layers, and to Buchta \& Freund (2017) for supersonic mixing layers. Unfortunately, very few computations of temporally-developing jets can be found in the literature, certainly because temporal jets do not exit from a nozzle, and have a potential core of infinite length, which renders the comparisons with spatially-developing jets difficult. Some have been carried out to examine the structure of the turbulence boundary of a planar jet (van Reeuwijk \& Holzner 2014) and to detect the presence of upstream-propagating waves in supersonic jets by (Bogey \& Gojon 2017), but to the best of the author's knowledge, the question of their sound field has never been addressed before. Therefore, it is unclear whether temporal jets radiate the same acoustic components as spatially-developing jets. If this is the case, however, original information on potential common sound sources could be gained when dealing with such flows.

In the present work, in order to provide new insight into the generation of noise in high-subsonic jet flows when the jet potential core closes, two temporally-developing isothermal round jets at a Mach number of 0.9 and diameter-based Reynolds numbers of 3,125 and 12,500 are considered. They are computed on a grid extending up to 120 jet radii in the axial direction by solving the compressible Navier-Stokes equations using high-order finite-difference schemes. The main statistical properties of the jet velocity and near pressure fields and velocity and pressure autocorrelations are calculated from ten runs carried out using different initial random perturbations in the jet shear layers. The first objective is to determine whether the two temporal jets generate sound waves in the same way as spatially-developing subsonic jets, including the jets of Stromberg et al. (1980), Freund (2001) and Bogey \& Bailly (2006, 2007) at low Reynolds numbers. The particular question that arises and needs to be answered here is whether, when the jet shear layers merge, low-frequency waves are emitted in the downstream direction as happens at the end of the potential core of spatial jets. If so, this will suggest that they are produced by the same noise generation mechanism, independently of the presence of a nozzle or of a potential core of finite length. The second objective is to find connections between these waves and the potential-core closing. With this aim in view, cross-correlations will be estimated between flow quantities and sound pressure outside the jet flow to track links between sources and observer. The third objective is to extract the the acoustic waves resulting from the potential-core closing from the background noise, and to reveal the corresponding noise generation mechanism in the flow. For this, conditional averages of the jet near fields will be calculated using a sample synchronization with salient flow events occurring during that closing. More precisely, these events will be detected when the minimum values of axial velocity on the jet axis due to the intermittent intrusion of turbulent structures in the jet are below an arbitrary threshold value.

The paper is organized as follows. The main characteristics of the two jets and of the simulations, including initial conditions, numerical methods, grid and computational parameters, are documented in section 2 . The simulation results, namely vorticity and pressure snapshots, the properties of the jet velocity and pressure fields, flow-noise crosscorrelations, and conditional samplings of the jet near fields are shown in section 3 . Concluding remarks are given in section 4 . Finally, the sound field of a temporal jet at a 
(a)

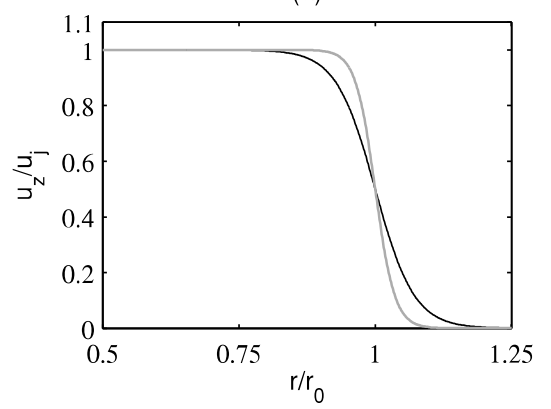

(b)

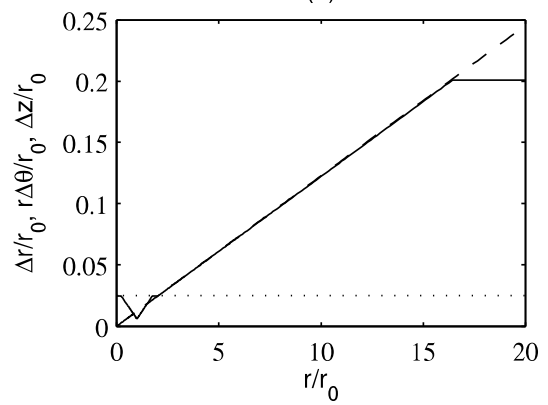

Figure 1. Radial profiles of (a) axial velocity $u_{z} / u_{j}$ at $t=0$ for $-\operatorname{Re}_{D}=3,125$ and $-\operatorname{Re}_{D}=12,500$, and (b) radial, azimuthal and axial mesh spacings $-\Delta r / r_{0}$, $--r \Delta \theta / r_{0}$ at $r=r_{0}$ and $-\cdot-\Delta z / r_{0}$.

Reynolds number of 50,000 is presented, and the influence of flow periodicity and of the number of runs on the statistical results and the choice of the threshold value used for the conditional sampling are discussed in four appendices.

\section{Parameters}

\subsection{Jet definition}

The two jets in this work are round and isothermal, and are characterized by a Mach number of $\mathrm{M}=u_{j} / c_{a}=0.9$ and Reynolds numbers of $\operatorname{Re}_{D}=u_{j} D / \nu_{a}=3,125$ and 12,500, where $u_{j}$ and $D=2 r_{0}$ are the jet initial centerline velocity and diameter, and $c_{a}$ and $\nu_{a}$ are the speed of sound and kinematic molecular viscosity in the ambient medium. The ambient temperature and pressure are $T_{a}=293 \mathrm{~K}$ and $p_{a}=10^{5} \mathrm{~Pa}$. At initial time $t=0$, the hyperbolic-tangent profiles of axial velocity presented in figure 1(a) are imposed. Following the variations of $\delta_{\theta} / r_{0}$ with the Reynolds number observed in experiments for initially laminar jets, e.g. in Zaman (1985), the velocity profile in the higher Reynolds number case is twice as thin. More precisely, the momentum thickness of the mixing layer is fixed at $\delta_{\theta}=0.0358 r_{0}$ for $\operatorname{Re}_{D}=3,125$ and $\delta_{\theta}=0.0179 r_{0}$ for $\operatorname{Re}_{D}=12,500$. This leads to initial momentum Reynolds numbers of $\operatorname{Re}_{\theta}=u_{j} \delta_{\theta} / \nu=56$ and 112, respectively. Radial and azimuthal velocities are set to zero, pressure is equal at $p_{a}$, and density is determined by a Crocco-Busemann relation.

At $t=0$, velocity perturbations of low amplitude are added in the mixing layers in order to seed the laminar-turbulent transition. For this, as proposed in Bogey et al. (2003), divergence-free Gaussian ring vortices of radius $r_{0}$ are imposed. These vortices have a half-width of $2 \delta_{\theta}$, and are regularly distributed in the axial direction every $\Delta z=0.025 r_{0}$, where $\Delta z$ is the axial mesh spacing. At each position, the vortex has a maximum velocity randomly fixed between 0 and $0.01 u_{j}$, and is weighted in the azimuthal direction by the function $\cos \left(n_{\theta} \theta+\varphi\right)$ where $n_{\theta}$ and $\varphi$ are randomly chosen between 0 and 32 and between 0 and $2 \pi$, respectively. This allows a peak rms value of velocity fluctuations of about $0.01 u_{j}$ to be obtained at $t=0$. Finally, note that ten runs are performed for each Reynolds number using different random seeds in order to improve the statistical convergence of the results.

The reasons for the choice of the Reynolds numbers of 3,125 and 12,500 are manyfold. First, it is possible to carry out very well-resolved numerical simulations of the two temporal jets on grids of large extent in the axial direction, where periodic conditions are applied and statistical averaging is performed. This is not the case for a jet at a 
Reynolds number of 50,000 for instance, as will be illustrated in appendix A. Second, according to experiments, jets at low Reynolds numbers, typically below $\operatorname{Re}_{D}=10^{5}$, are initially laminar (Crighton 1981; Zaman 1985). Therefore, there does not appear necessary to impose a boundary-layer profile at $t=0$, which would be difficult in the present simulations because of the absence of a nozzle. Third, the downstream noise component of subsonic jets is expected to persist at low Reynolds numbers (Stromberg et al. 1980; Kastner et al. 2006; Bogey \& Bailly 2007). It may even be more predominant than at high Reynolds numbers, which should help us to extract its associated generation mechanism.

\subsection{Numerical methods}

The numerical framework is identical to that used in recent simulations of round jets (Bogey \& Bailly 2010; Bogey et al. 2012, 2011b; Bogey \& Marsden 2016). The simulations have been carried out using an in-house solver of the three-dimensional filtered compressible Navier-Stokes equations in cylindrical coordinates $(r, \theta, z)$ based on low-dissipation and low-dispersion, high-order explicit schemes. The axis singularity is taken into account by the method of Mohseni \& Colonius (2000). In order to alleviate the time-step restriction near the cylindrical origin, the derivatives in the azimuthal direction around the axis are calculated at coarser resolutions than permitted by the grid (Bogey et al. $2011 a$ ). For the points closest to the jet axis, they are evaluated using 16 points, yielding an effective resolution of $2 \pi / 16$. Fourth-order eleven-point centered finite differences are used for spatial discretization, and a second-order six-stage Runge-Kutta algorithm is implemented for time integration (Bogey \& Bailly 2004). A twelfth-order eleven-point centered filter is applied explicitly to the flow variables every time step in order to remove grid-to-grid oscillations while leaving larger scales mostly unaffected. Non-centered finite differences and filters are also used near the grid boundaries (Berland et al. 2007; Bogey \& Bailly 2010). The radiation conditions of Tam \& Dong (1996), extended in 3-D in Bogey \& Bailly (2002), are applied at the sideline boundaries to avoid significant acoustic reflections. Since a temporally-developing flow is considered, periodic boundary conditions are imposed in the axial direction. Given the size of the computation domain in that direction, as shown in appendix B providing velocity correlation functions in the jets at $r=0$ and $r=r_{0}$ at different times, these conditions do not appear to distort the jet flow development.

\subsection{Simulation parameters}

The mesh grid used in the different runs extends up to $z=120 r_{0}$ in the axial direction, and out to $r=30 r_{0}$ in the radial direction. It contains $n_{r} \times n_{\theta} \times n_{z}=382 \times 512 \times 4,800=$ 940 million points. The mesh spacing in the axial direction is uniform and equal to $\Delta z=0.025 r_{0}$, whereas, as illustrated in figure $1(\mathrm{~b})$, the mesh spacing in the radial direction varies significantly. The latter is equal to a minimum value of $\Delta r=0.006 r_{0}$ at $r=r_{0}$, and to $\Delta r=0.025 r_{0}=\Delta z$ on the jet axis. It is maximum and equal to $\Delta r=0.2 r_{0}$ for $r \geq 16 r_{0}$, yielding a Strouhal number of $\mathrm{St}_{D}=2.8$ for an acoustic wave discretized by four points per wavelength. The use of $n_{\theta}=512$ points in the azimuthal direction leads to the azimuthal mesh spacings of $r \Delta \theta=0.012 r_{0}$ at $r=r_{0}$ and of $r \Delta \theta=0.025 r_{0}=\Delta z$ at $r=2 r_{0}$. Furthermore, the simulations have been checked, from the calculation of the turbulent kinetic energy budgets, to be fully resolved direct numerical simulations for the jet at $\operatorname{Re}_{D}=3,125$ and very well resolved large-eddy simulations for the jet at $\operatorname{Re}_{D}=12,500$.

The computations have been performed using an OpenMP-based in-house solver on 16-core and 32-core nodes of Intel Xeon E5 processors. In order to ensure the stability of 
the explicit time advancement, the time step $\Delta t$ is chosen so that $\Delta t=0.6 \Delta r\left(r=r_{0}\right) / c_{a}$ for the jet at $\operatorname{Re}_{D}=3,125$, and $\Delta t=\Delta r\left(r=r_{0}\right) / c_{a}$ for $\operatorname{Re}_{D}=12,500$. Its lowering as the Reynolds number decreases is due to the integration of the viscous fluxes. The total number of iterations is equal to 22,600 for $\operatorname{Re}_{D}=3,125$ and to 13,600 for $\operatorname{Re}_{D}=12,500$, to reach a final time of $t=75 r_{0} / u_{j}$ in both cases. During the simulations, density, the three velocity components, pressure and vorticity norm are recorded on the jet axis at $r=0$ and on the cylindrical surfaces at $r=r_{0}, 4 r_{0}$ and $20 r_{0}$, at a sampling frequency allowing time spectra to be computed up to $\mathrm{St}_{D}=10$, and on the four azimuthal planes at $\theta=0, \pi / 2, \pi$ and $3 \pi / 2$, at half the frequency mentioned above. The statistical results obtained in each run are averaged over the periodic directions $z$ and $\theta$. For both jets, the results of the different runs are then ensemble averaged, providing mean values, denoted by $<$. $>$ in what follows, calculated over an equivalent distance of $1,200 r_{0}$ in the streamwise direction. Thus, the statistics can be considered to be well converged. This is demonstrated in appendix C comparing results, namely mean centerline velocities, peak turbulence intensities, and rms values and spectra of pressure fluctuations, obtained from 5 and 10 runs for the two jets.

\section{Results}

\subsection{Vorticity and pressure snapshots}

Snapshots of the vorticity norm and pressure fluctuations obtained in the $(z, r)$ plane for the jets at $\operatorname{Re}_{D}=3,125$ and $\operatorname{Re}_{D}=12,500$ are represented, respectively, in figure 2 at times $t u_{j} / r_{0}=15,25,35$ and 45 and in figure 3 at $t u_{j} / r_{0}=10,20,30$ and 40 , and in the corresponding movies movie_fig2 and movie_fig3 available at journals.cambridge.org/flm.

For both Reynolds numbers, two mixing layers can be seen on both sides of the jet axis in the top pictures. They have just rolled up following the growth of instability waves in the hyperbolic-tangent velocity profile (Michalke 1964) for $\operatorname{Re}_{D}=3,125$, whereas they appear to be turbulent for $\operatorname{Re}_{D}=12,500$. In the second pictures from top, the mixing layers, which have developed thanks to the vortex-pairing mechanism (Winant \& Browand 1974), merge on the jet axis, resulting in the disappearance of the potential core. Finally, in the third and fourth pictures from top, the jets are fully developed, and contain vortical scales of intensity decreasing with time. Furthermore, more fine turbulent scales are observed in the higher Reynolds number jet, as expected.

For $\operatorname{Re}_{D}=3,125$, in figure 2 , alternatively positive and negative fluctuations are found at $t u_{j} / r_{0}=15$ in the pressure field in the close vicinity of the jet. They are most likely the footprints of the coherent turbulent structures of the jet flow in the near field (Arndt et al. 1997; Coiffet et al. 2005). At $t u_{j} / r_{0}=25$, as the potential core closes, high-amplitude waves are emitted in the downstream direction. These waves are well visible at later times $t u_{j} / r_{0}=35$ and 45 . They appear to be symmetric with respect to the jet centerline and to have a typical wavelength of about $15 r_{0}$. Moreover, they propagate mainly in the downstream direction, and have a very large spatial extent along the wave front direction. Interestingly, they look like the waves emitted at shallow angles by spatially-developing subsonic jets at both low and high Reynolds numbers, see for instance the simulation results in Bogey \& Bailly (2006) and Bogey (2018).

For $\operatorname{Re}_{D}=12,500$, in figure 3 , the pressure field exhibits, unsurprisingly, more waves of short wavelength than previously for $\operatorname{Re}_{D}=3,125$, but also additional noise components, whose contribution will be later quantified in the pressure spectra of figure 14 . In particular, sound waves generated by vortex pairings in the mixing layers are noted 
at $t u_{j} / r_{0}=10$ and 20 . In addition, upstream-propagating high-frequency waves are detected at $t u_{j} / r_{0}=30$ and 40 after the potential-core closing, which was not the case for the first jet. At these two times, more importantly given the aim of the present study, low-frequency waves are found to propagate in the downstream direction. Even at a lower level, they resemble those obtained for the jet at $\operatorname{Re}_{D}=3,125$. Similar acoustic waves of comparable amplitude also appear to be radiated by a temporal jet at $\operatorname{Re}_{D}=50,000$, computed in an extra simulation reported in appendix A. Therefore, these downstreampropagating sound waves, as well as their corresponding noise generation mechanism, persist when the Reynolds number increases, despite the richer spectral content of the jet turbulence, hence the relative weakening of the flow large-scale structures, in that case. Furthermore, they seem not to depend much on the shear-layer initial momentum thickness given that at $t=0$, one sets $\delta_{\theta}=0.0358 r_{0}$ for $\operatorname{Re}_{D}=3,125, \delta_{\theta}=0.0179 r_{0}$ for $\operatorname{Re}_{D}=12,500$ and $\delta_{\theta}=0.0089 r_{0}$ for $\operatorname{Re}_{D}=50,000$.

\subsection{Properties of the velocity fields}

\subsubsection{Velocity field statistics}

The mean and rms values of axial velocity calculated for the jets are represented in figure 4 using $(t, r)$ coordinates. The results bear striking similarities with the flow fields measured in the $(z, r)$ plane of spatially-developing subsonic jets, by Zaman (1986) for example. The mean axial velocity fields of figures $4(a, b)$ show the jet development with time, and indicates that the potential core closes at $t u_{j} / r_{0} \simeq 21$ for $\operatorname{Re}_{D}=3,135$ and earlier at $t u_{j} / r_{0} \simeq 16$ for $\operatorname{Re}_{D}=12,500$. In parallel, the axial turbulence intensities are found in figure $4(\mathrm{c}, \mathrm{d})$ to grow in the jet shear layer, to reach values slightly higher than $20 \%$, and then to decrease. The peak values are obtained around $t u_{j} / r_{0}=21$ for $\operatorname{Re}_{D}=3,135$ and $t u_{j} / r_{0}=8$ for $\operatorname{Re}_{D}=12,500$, that is near the time of potential-core closing in the first case, but significantly before in the second case.

The time variations of the shear-layer momentum thickness, calculated here as

$$
\delta_{\theta}=\int_{0}^{r_{0.001}} \frac{<u_{z}>}{\left.<u_{z}\right\rangle(r=0)}\left(1-\frac{\left.<u_{z}\right\rangle}{<u_{z}>(r=0)}\right) d r
$$

where $r_{0.001}$ is such that $\left.\left\langle u_{z}\right\rangle\left(r=r_{0.001}\right)=0.001 \times<u_{z}\right\rangle(r=0)$, and of the mean centerline axial velocity are plotted in figure 5 . In figure $5(\mathrm{a})$, the shear layers appear to spread slowly when the jet flows are laminar or turbulent, but more rapidly during the laminar-turbulent transitional periods, which happen between $t u_{j} / r_{0} \simeq 12.5$ and 25 for $\operatorname{Re}_{D}=3,125$, and between $t u_{j} / r_{0} \simeq 5$ and 12.5 for $\operatorname{Re}_{D}=12,500$. In figure $5(\mathrm{~b})$, the velocity decay after the potential-core closing starts at $t_{c} u_{j} / r_{0}=21.6$ for $\operatorname{Re}_{D}=3,125$ and at $t_{c} u_{j} / r_{0}=16$ for $\operatorname{Re}_{D}=12,500$, where $t_{c}$ is arbitrarily defined by $\left\langle u_{z}(r=0, t=\right.$ $\left.\left.t_{c}\right)\right\rangle=0.95 u_{j}$. Afterwards, the decay occurs at a higher rate in the lower Reynolds number case.

The time variations of the rms values and of the skewness factors of axial velocity fluctuations at $r=0$ and $r=r_{0}$ are displayed in figure 6. In figure 6(a), strong humps are obtained in the profiles of turbulence intensity, leading to maximum values of $17 \%$ at $r=0$ and $20 \%$ at $r=r_{0}$ for $\operatorname{Re}_{D}=3,125$, and of $12.6 \%$ at $r=0$ and $21.3 \%$ at at $r=r_{0}$ for $\operatorname{Re}_{D}=12,500$. They result from the mergings of vortical structures in the shear layers and on the jet centerline, respectively (Bogey \& Bailly 2010). As expected, due to the difference in mean flow development, they occur more rapidly in the $\operatorname{Re}_{D}=12,500$ case. Moreover, the peak value at $r=r_{0}$ is reached much earlier than the peak value at $r=0$ for $\operatorname{Re}_{D}=12,500$, but that is not true for $\operatorname{Re}_{D}=3,125$. This suggests the presence of vortex pairings in the mixing layers only in the higher Reynolds number jet.

In figures $6(\mathrm{~b})$, values of skewness around -1.1 are found at $r=0$ close to the time of 

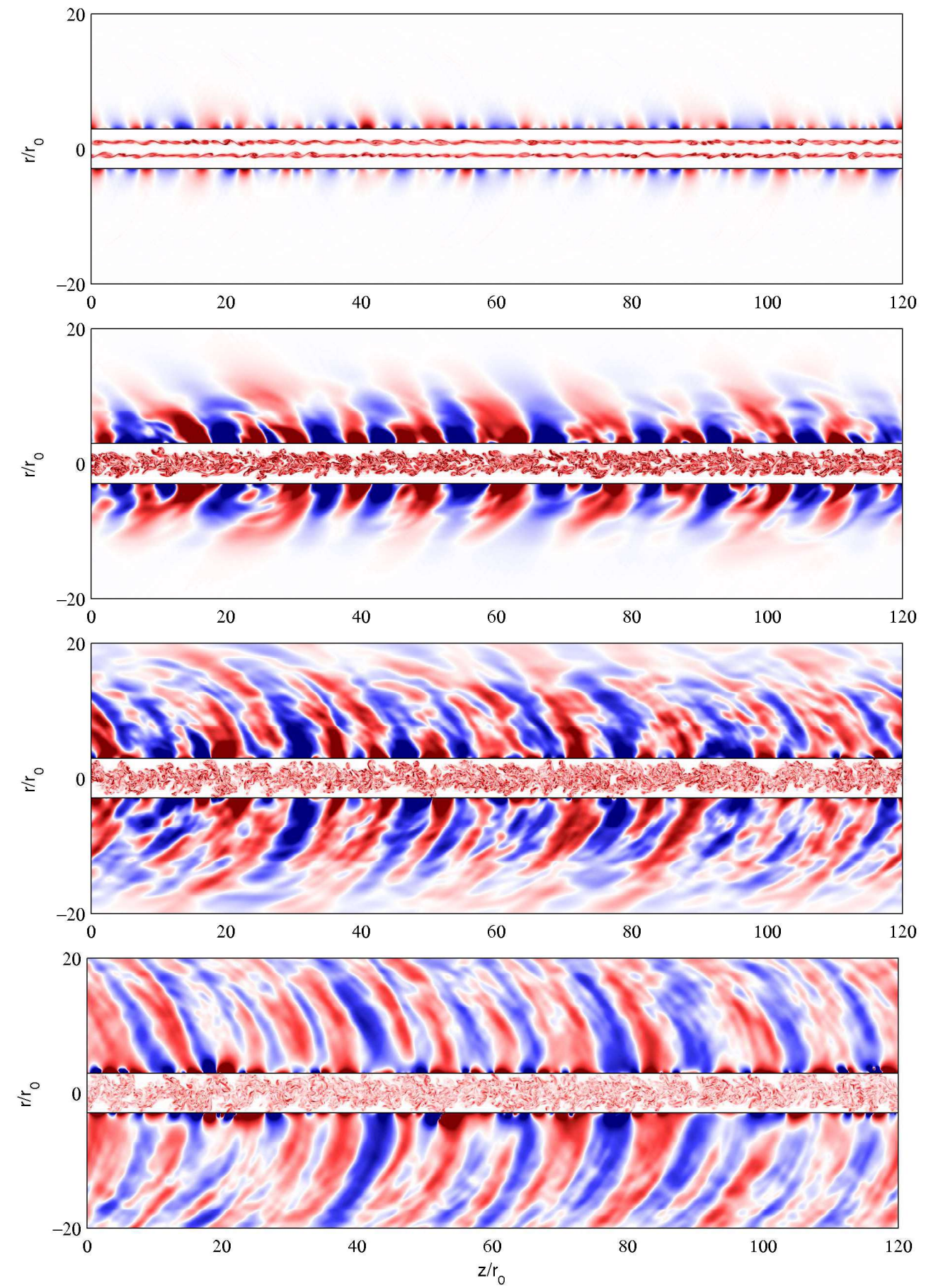

FiguRE 2. (Colour and movie online available at journals . cambridge.org/flm) Representation of vorticity norm for $r \leq 3 r_{0}$ and of pressure fluctuations otherwise, obtained for $\operatorname{Re}_{D}=3,125$ at $t u_{j} / r_{0}=15,25,35$ and 45, from top to bottom. The color scales range up to the level of $5 u_{j} / r_{0}$ for vorticity, and from $-250 \mathrm{~Pa}$ to $250 \mathrm{~Pa}$ for pressure, from blue to red. 

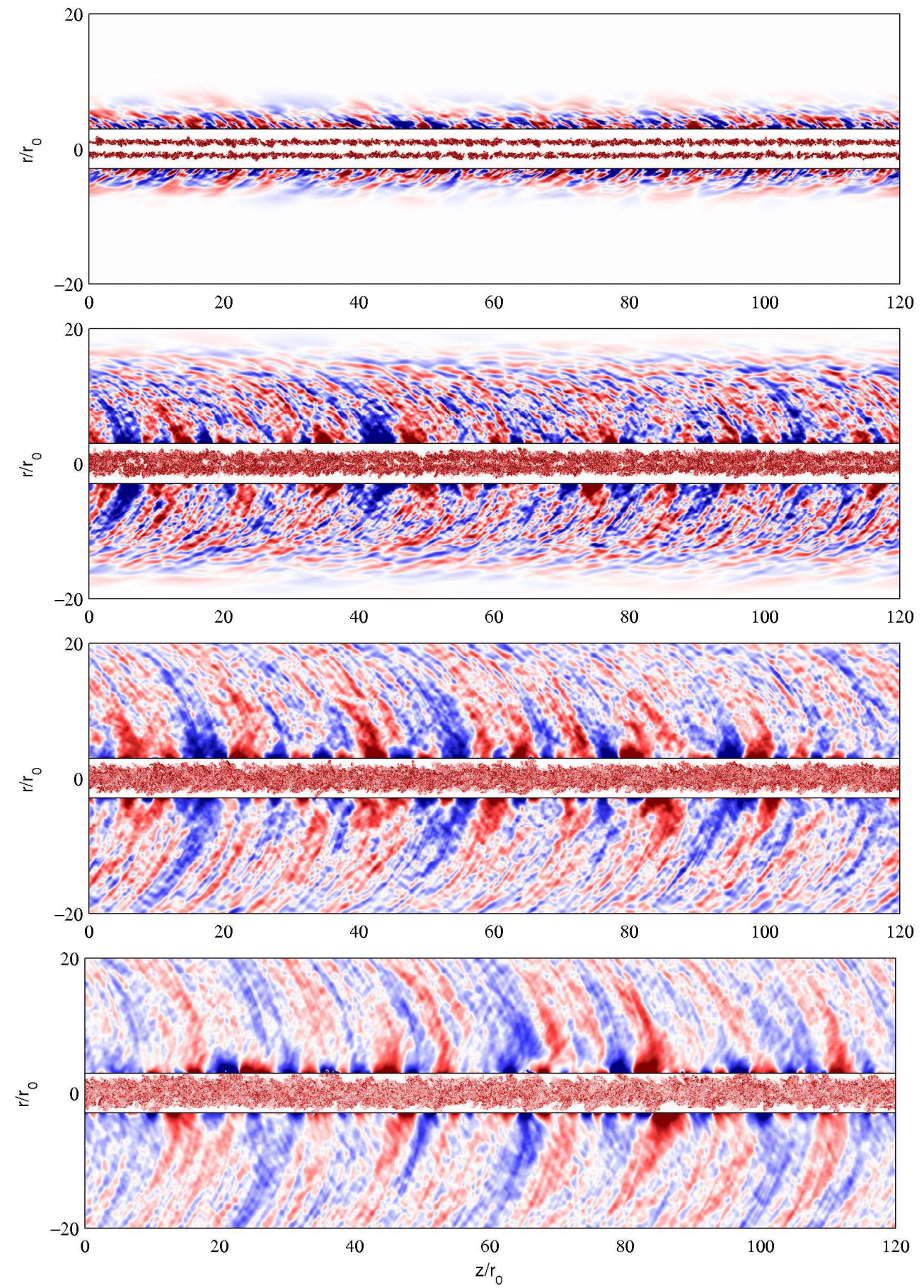

Figure 3. (Colour and movie online available at journals . cambridge.org/flm) Representation of vorticity norm for $r \leq 3 r_{0}$ and of pressure fluctuations otherwise, obtained for $\operatorname{Re}_{D}=12,500$ at $t u_{j} / r_{0}=10,20,30$ and 40, from top to bottom. The color scales range up to the level of $5 u_{j} / r_{0}$ for vorticity, and from $-200 \mathrm{~Pa}$ to $200 \mathrm{~Pa}$ for pressure, from blue to red. 
(a)

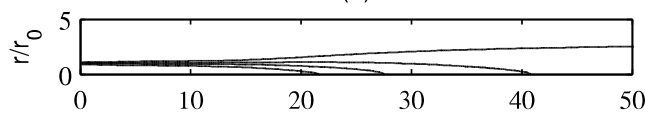

(c)

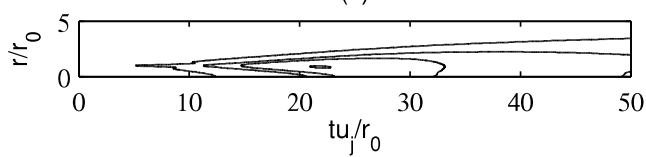

(b)

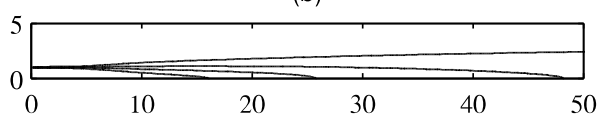

(d)

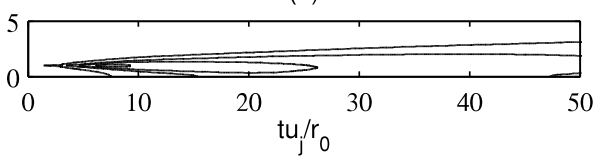

Figure 4. Space-time representation of (top) mean and (bottom) rms fields of axial velocity for (left) $\operatorname{Re}_{D}=3,125$ and (right) $\operatorname{Re}_{D}=12,500$ using the contour line values: $(\mathrm{a}, \mathrm{b})\left\langle u_{z}\right\rangle / u_{j}=0.05$, $0.35,0.65$ and $0.95,(\mathrm{c}, \mathrm{d})\left\langle u_{z}^{\prime} u_{z}^{\prime}\right\rangle^{1 / 2} / u_{j}=0.02,0.08,0.14$ and 0.20 .

(a)

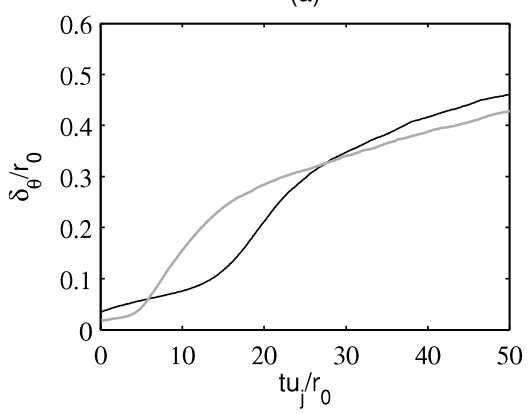

(b)

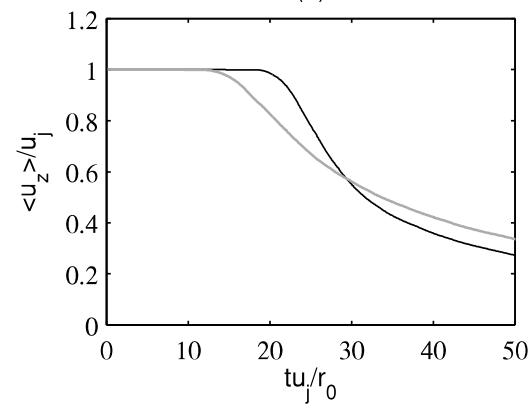

Figure 5. Time variations of (a) shear-layer momentum thickness $\delta_{\theta} / r_{0}$ and (b) mean axial velocity $\left\langle u_{z}\right\rangle / u_{j}$ at $r=0$ for $\longrightarrow \operatorname{Re}_{D}=3,125$ and $\longrightarrow \operatorname{Re}_{D}=12,500$.

(a)

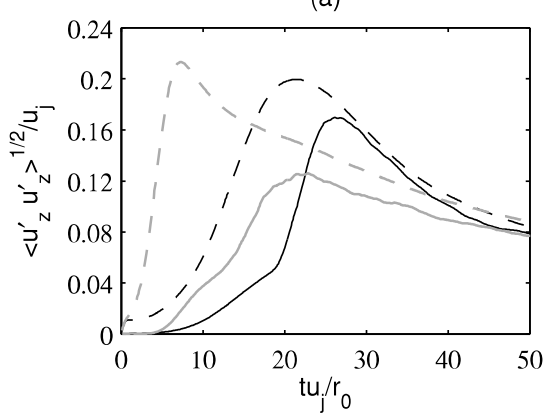

(b)

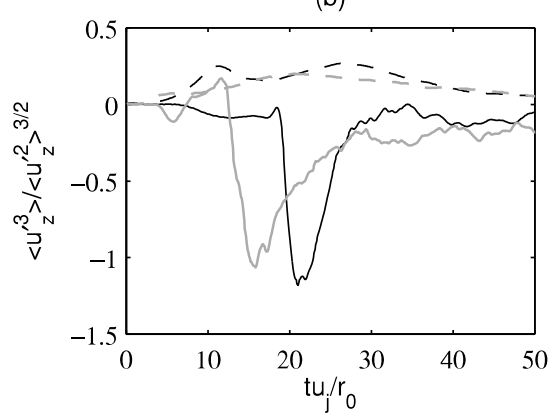

Figure 6. Time variations of (a) axial turbulence intensity $\left\langle u_{z}^{\prime} u_{z}^{\prime}\right\rangle^{1 / 2} / u_{j}$ and (b) the skewness factor of axial velocity fluctuations $u_{z}^{\prime}$ : at $r=0$ for $-\operatorname{Re}_{D}=3,125$ and $\operatorname{Re}_{D}=12,500$, and at $r=r_{0}$ for $---\operatorname{Re}_{D}=3,125$ and $--\operatorname{Re}_{D}=12,500$.

potential-core closing for both Reynolds numbers. This indicates intermittent occurrence of velocity deficits on the jet centerline at that time. These deficits follow the intrusion of shear-layer turbulent structures in the potential core of the temporal jet, as it happens at the end of the potential core of spatially-developing jets (Bogey et al. 2003; Bogey \& Bailly 2007). At $r=r_{0}$, the results are quite different from those at $r=0$. In this case, the skewness factor is slightly positive, suggesting possible bursts of high-velocity flow structures in the mixing layers. 
(a)

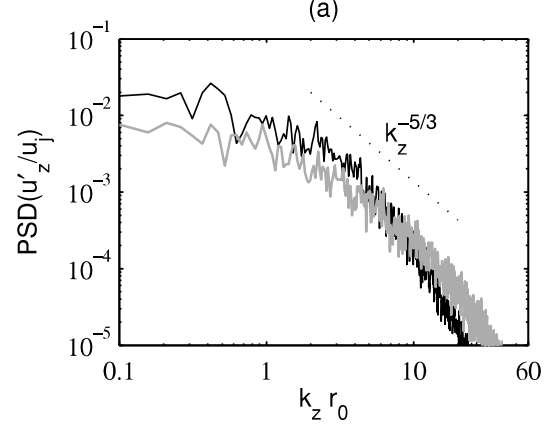

(b)

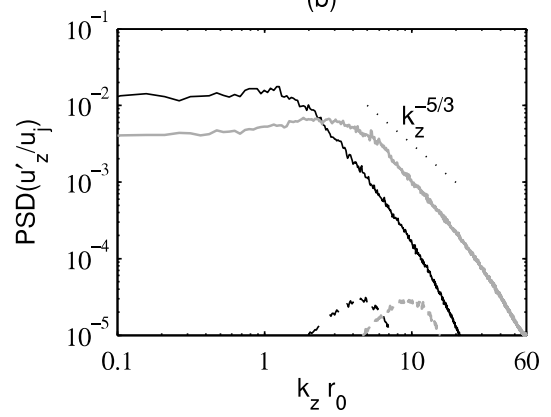

Figure 7 . Representation of the power spectral densities of axial velocity fluctuations $u_{z}^{\prime} / u_{j}$ as a function of axial wavenumber $k_{z} r_{0}$ : (a) at $r=0$ at time of peak level for $-\operatorname{Re}_{D}=3,125$ and $-\operatorname{Re}_{D}=12,500$, (b) at $r=r_{0}$ at time of peak level for $-\operatorname{Re}_{D}=3,125$ and $-\operatorname{Re}_{D}=12,500$, and at $t u_{j} / r_{0}=2$ for $---\operatorname{Re}_{D}=3,125$ and $t u_{j} / r_{0}=1$ for -$-\operatorname{Re}_{D}=12,500 ;-\cdot-\cdot k_{z}^{-5 / 3}$ slope.

\subsubsection{Velocity spectra}

Spectra of axial velocity fluctuations computed in the jet flows at $r=0$ and $r=r_{0}$ are represented in figure 7 as a function of normalized axial wavenumber $k_{z} r_{0}$, up to the value of $k_{z} r_{0}=60$ which is close to the filtering cut-off wave number of $k_{z} r_{0}=62.8$ obtained for four points per wavelength. The spectra calculated on the jet axis at the times of peak turbulence intensity level $t u_{j} / r_{0}=26.2$ for $\operatorname{Re}_{D}=3,125$ and $t u_{j} / r_{0}=22.8$ for $\operatorname{Re}_{D}=12,500$, just after the potential-core closing, are depicted in figure $7(\mathrm{a})$. They have similar shapes, which are consistent with the $k_{z}^{-5 / 3}$ power law for $k_{z} r_{0} \geq 2$. However, the spectrum for $\operatorname{Re}_{D}=3,125$ contains stronger low-frequency components than that for $\operatorname{Re}_{D}=12,500$. It seems also to be dominated by components around $k_{z} r_{0} \simeq 0.4$, corresponding to a wavelength of $\lambda_{z} \simeq 15 r_{0}$, which is not the case for the higher Reynolds number jet.

The velocity spectra estimated at $r=r_{0}$ are shown in figure $7(\mathrm{~b})$. At the early times of $t u_{j} / r_{0}=2$ for $\operatorname{Re}_{D}=3,125$ and $t u_{j} / r_{0}=1$ for $\operatorname{Re}_{D}=12,500$, components are found to emerge around wave numbers $k_{z} r_{0}=4.8$ and 10.2 , respectively. These peak wave numbers are slightly lower than those given by the inviscid linear stability theory of Michalke (1964), predicting that the amplification of temporally-growing instability waves is maximum at $k_{z} r_{0}=6.2$ and 12.4 in the two hyperbolic-tangent velocity profiles imposed at $t=0$. The discrepancy between computations and theory is likely due to the initial conditions, but also to the low Reynolds numbers of $\operatorname{Re}_{\theta}=56$ and 112 in the simulated shear layers. For such values, indeed, the growth rates and the peak wave numbers of instability waves can be expected to be reduced compared to the inviscid case, as reported in the theoretical analyses conducted by Ragab \& Wu (1989) for twodimensional mixing layers and by Morris (1976) for axisymmetric jets.

As for the velocity spectra obtained at $r=r_{0}$ at the times of peak turbulence intensity level $t u_{j} / r_{0}=21.6$ for $\operatorname{Re}_{D}=3,125$ and $t u_{j} / r_{0}=7.3$ for $\operatorname{Re}_{D}=12,500$, also plotted in figure $7(\mathrm{~b})$, they are relatively broadband, and show no clear agreement with the $k_{z}^{-5 / 3}$ power law. In this case, the dominant components are noted around $k_{z} r_{0}=1$ for $\operatorname{Re}_{D}=3,125$ and $k_{z} r_{0}=2.4$ for $\operatorname{Re}_{D}=12,500$. The difference in peak wave number can be explained by the fact that the maximum turbulence level is reached at the time of potential-core closing for the first jet, but much earlier, in the developing mixing layers, for the other jet. 

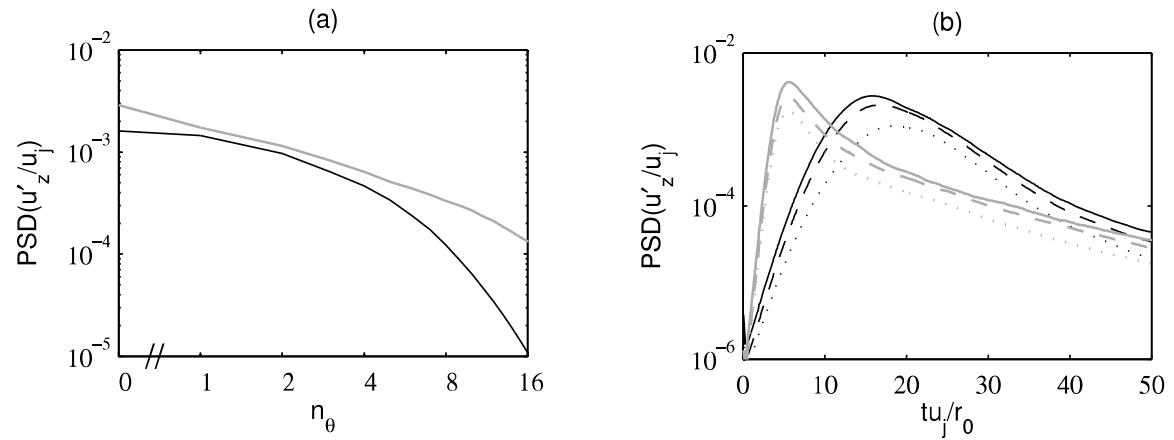

FiguRE 8. Representation of the power spectral densities of axial velocity fluctuations $u_{z}^{\prime} / u_{j}$ obtained at $r=r_{0}$ : (a) at time of peak level for $-\operatorname{Re}_{D}=3,125$ and $\longrightarrow \operatorname{Re}_{D}=12,500$ as a function of azimuthal mode $n_{\theta}$, (b) time variations of the magnitudes of modes $n_{\theta}=0,---n_{\theta}=1$ and $-\cdot-\cdot n_{\theta}=2$ for $\operatorname{Re}_{D}=3,125$, and $-n_{\theta}=0,---$ $n_{\theta}=1$ and $-\cdots n_{\theta}=2$ for $\operatorname{Re}_{D}=12,500$.

In order to explore the coherence of the flow field in the azimuthal direction, the velocity spectra obtained at $r=r_{0}$ at the times of peak turbulence intensity level are displayed in figure $8(\mathrm{a})$ as a function of azimuthal mode $n_{\theta}$. The time variations of the magnitudes of modes $n_{\theta}=0,1$ and 2 at $r=r_{0}$ are also represented in figure 8(b). For both jets, at all times, the contribution of the axisymmetric mode is dominant, closely followed by the contribution of mode $n_{\theta}=1$. This is in line with the results of the literature about the amplification rates of spatially-growing linear instability waves in jet flows, see for example in Michalke (1984) for the inviscid incompressible case and in Keiderling et al. (2009) for a viscous jet at a Mach number of 0.9 .

\subsubsection{Velocity autocorrelations}

In the jet flow field, the space-time autocorrelations of axial velocity fluctuations are computed at radial position $r$ and time $t$ in the following way

$$
\mathcal{R}_{u_{z}^{\prime} u_{z}^{\prime}}(\delta z, \delta t)=\frac{\left\langle u_{z}^{\prime}(r, \theta, z, t) u_{z}^{\prime}(r, \theta, z+\delta z, t+\delta t)\right\rangle}{\left\langle u_{z}^{\prime 2}(r, \theta, z, t)\right\rangle^{1 / 2}\left\langle u_{z}^{\prime 2}(r, \theta, z+\delta z, t+\delta t)\right\rangle^{1 / 2}}
$$

where $\delta z$ is the spatial separation in the axial direction and $\delta t$ is the time delay.

The correlation maps obtained on the jet centerline are represented in figure 9 at times $t=t_{c}-6 r_{0} / u_{j}, t=t_{c}$ and $t=t_{c}+6 r_{0} / u_{j}$, and in the corresponding movies movie_fig9a and movie_fig9b available at journals.cambridge.org/flm. The solid and dashed lines show the inclinations expected for convection velocities of $0.6 u_{j}$ and $u_{j}$. The results for the two jets are similar. In figures $9(\mathrm{a}, \mathrm{d})$, the correlations at $t=t_{c}-6 r_{0} / u_{j}$ before the potential-core closing have an oscillating shape in the axial direction, and remain significant for very large time delays. In addition, they are well aligned with the trajectory for a convection velocity of $0.6 u_{j}$. In figures $9(\mathrm{~b}, \mathrm{e})$, the correlations at $t=t_{c}$ are comparable to the previous ones for negative time delays, but differ for positive time delays. In the latter case, the correlations are only positive and their inclination gets closer to that corresponding to a convection velocity of $u_{j}$. This is due to the arrival of turbulent structures on the centerline when the potential core closes. Finally, the correlations at later times become less inclined with increasing time, as the jets develop and the velocity decays on the centerline.

For comparison, the correlation maps obtained at $r=r_{0}$ are provided in figure 10 . They resemble the correlations at $r=0$ given in figure 9 at the same times. Slight 
(a)

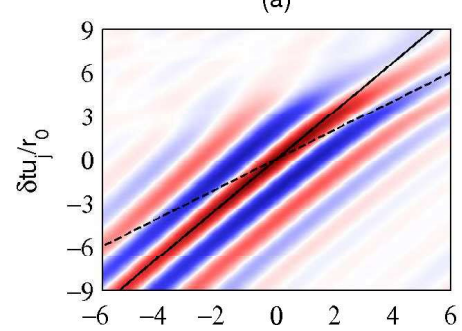

(d)

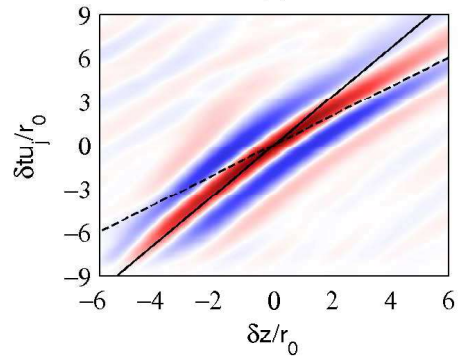

(b)

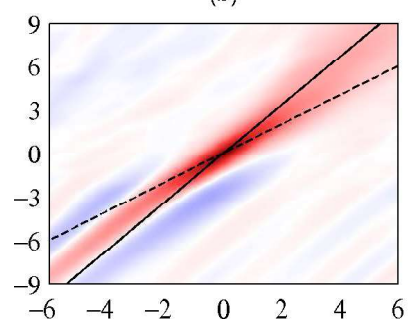

(e)

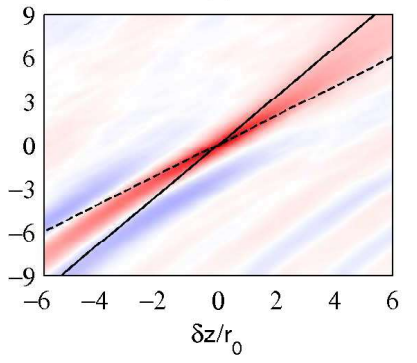

(c)

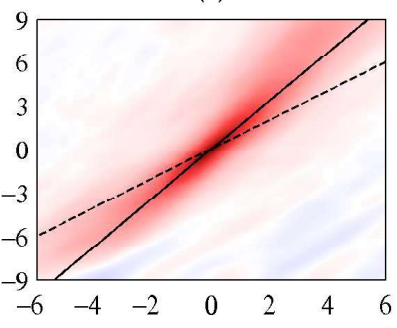

(f)

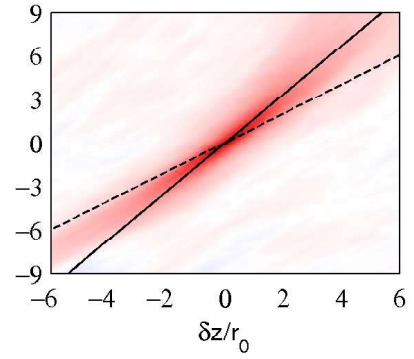

FIgURE 9. (Colour and movie available at journals. cambridge.org/flm) Representation of the space-time correlations of axial velocity fluctuations obtained at $r=0$ at (a,d) $t=t_{c}-6 r_{0} / u_{j}$, (b,e) $t=t_{c}$ and (c,f) $t=t_{c}+6 r_{0} / u_{j}$ for (top) $\operatorname{Re}_{D}=3,125$ and (bottom) $\operatorname{Re}_{D}=12,500$; to red.

differences however appear. First, before the potential-core closing, significant correlation levels persist for shorter spatial separations and time delays. This may result from the fact that for $t<t_{c}$, the correlations on the centerline are calculated from the footprints of the coherent structures developing in the mixing layers, whereas those at $r=r_{0}$ are computed from a wider variety of turbulent scales. Second, the trajectories of the correlation spots are less inclined. This is particularly true at $t=t_{c}$, where they indicate a convection velocity lower than $0.6 u_{j}$.

Some flow properties estimated from the velocity correlation functions, namely the integral length scale $L_{u u}^{(z)}$ and the convection velocity $u_{c}$, are shown in figure 11. In figure 11(a), the integral length scales are found to increase with time. The increase occurs suddenly around the time of potential-core closing on the jet axis, but progressively at $r=r_{0}$. Furthermore, at $t>t_{c}$, the integral length scales are larger for $\operatorname{Re}_{D}=3,125$ than for $\operatorname{Re}_{D}=12,500$, and on the jet axis than at $r=r_{0}$. In figure $11(\mathrm{~b})$, the convection velocities at $r=r_{0}$ decrease monotonically with time from $u_{c} \simeq 0.6 u_{j}$ at $t u_{j} / r_{0}=10$ down to $u_{c} \simeq 0.3 u_{j}$ at $t u_{j} / r_{0}=50$. On the jet axis, on the contrary, they first increase, then reach peak values of $0.86 u_{j}$ for $\operatorname{Re}_{D}=3125$ and of $0.95 u_{j}$ for $\operatorname{Re}_{D}=12500$ near the time of potential-core closing, and finally diminish. This suggests that the turbulent structures that enter in the potential core are convected at a velocity close to the jet velocity, as in spatially-developing jets (Bogey \& Bailly 2007).

\subsection{Properties of the pressure fields}

\subsubsection{Pressure field statistics}

The rms values of pressure fluctuations obtained for the jets are represented in figure 12 using $(t, r)$ coordinates. As previously for the velocity, the results resemble the rms pressure fields acquired in the $(z, r)$ plane of spatially-developing subsonic jets (Zaman 1986; 
(a)

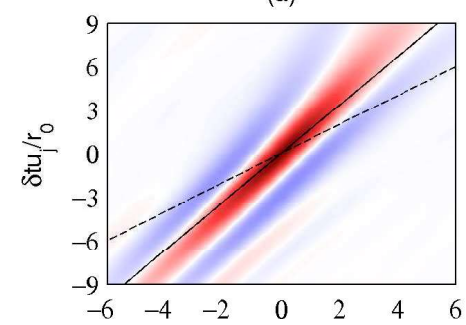

(d)

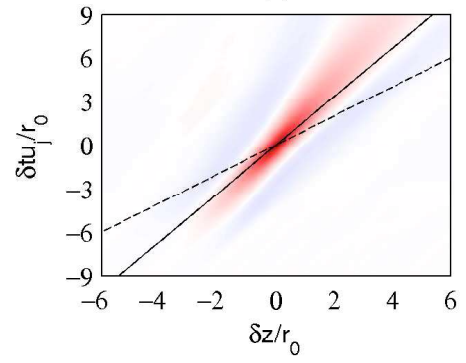

(b)

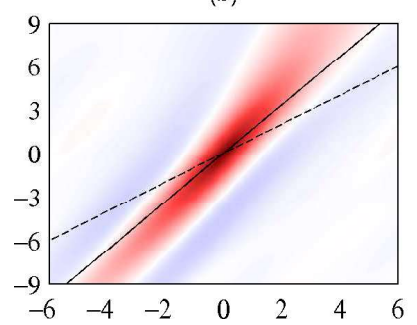

(e)

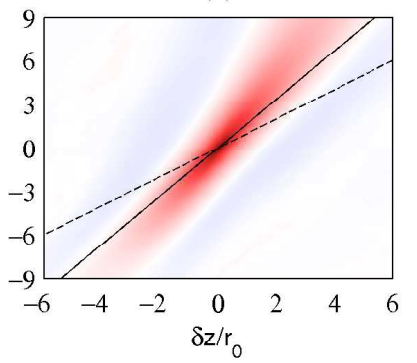

(c)

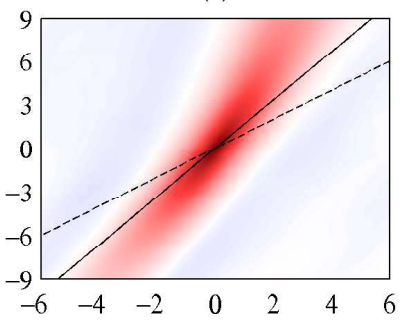

(f)

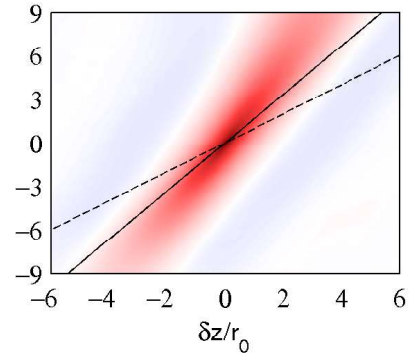

Figure 10. (Colour available and movies online available at journals.cambridge.org/flm) Representation of the space-time correlations of axial velocity fluctuations obtained at $r=r_{0}$ at (a,d) $t=t_{c}-6 r_{0} / u_{j}$, (b,e) $t=t_{c}$ and (c,f) $t=t_{c}+6 r_{0} / u_{j}$ for (top) $\operatorname{Re}_{D}=3,125$ and (bottom) $\operatorname{Re}_{D}=12,500 ;-\delta t=\delta z /\left(0.6 u_{j}\right) ;---\delta t=\delta z / u_{j}$. The color scale ranges from -1 to 1 , from blue to red.

(a)

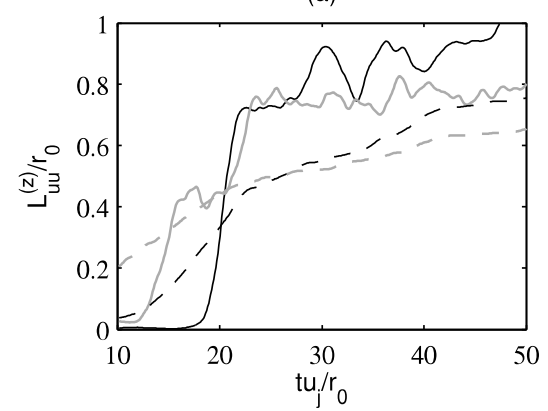

(b)

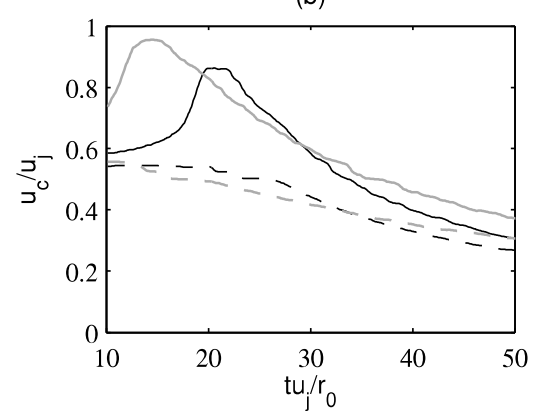

Figure 11. Time variations of (a) the integral length scale $L_{u u}^{(z)} / r_{0}$ and (b) the convection velocity $u_{c} / u_{j}$, obtained at $r=0$ for $-\operatorname{Re}_{D}=3,125$ and $\longrightarrow \operatorname{Re}_{D}=12,500$, and at $r=r_{0}$ for $---\operatorname{Re}_{D}=3,125$ and $--\operatorname{Re}_{D}=12,500$.

Ukeiley \& Ponton 2004; Bogey et al. 2007). For both Reynolds numbers, the maximum values are located in the jet flows, approximately at the same position as the maximum rms values of axial velocity in figure $5(\mathrm{c}, \mathrm{d})$. Outside the jets, lobes, whose origins seem to be the peak levels in the flow (Zaman 1986), are found. They reveal the production of noise in the jet and its propagation outside with increasing time. The rms pressure fields moreover suggest that the strongest acoustic waves are generated close to the time of potential-core closing for $\operatorname{Re}_{D}=3,125$, but well before for $\operatorname{Re}_{D}=12,500$, and that their main radiation angle with respect to the flow direction is larger in the latter case than in the former.

The time variations of the rms values of pressure fluctuations at $r=10 r_{0}$ are shown in figure $13(\mathrm{a})$. The profiles reach maximum values of $83 \mathrm{~Pa}$ at $t u_{j} / r_{0}=35.4$ for $\mathrm{Re}_{D}=$ 
(a)

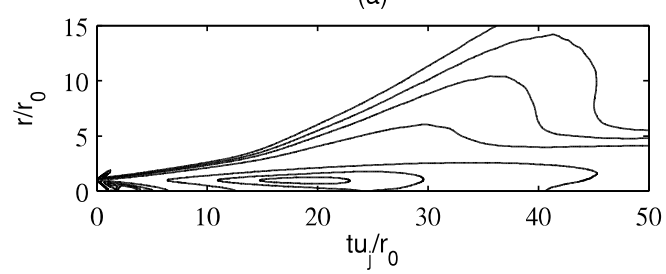

(b)

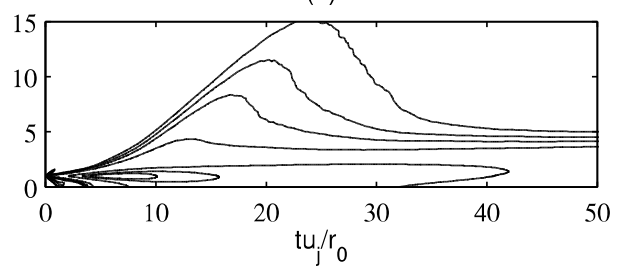

FIgURE 12. Space-time representation of the rms fields of pressure fluctuations for (a) $\operatorname{Re}_{D}=3,125$ and (b) $\operatorname{Re}_{D}=12,500$ using the contour line values $\left\langle p^{\prime} p^{\prime}\right\rangle^{1 / 2}=45,60$, $80,140,1000,3000$ and $5000 \mathrm{~Pa}$.

(a)

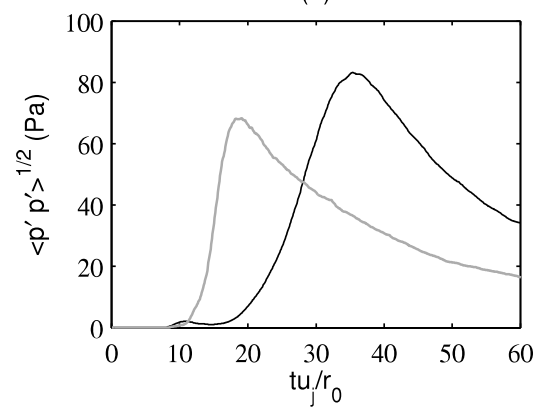

(b)

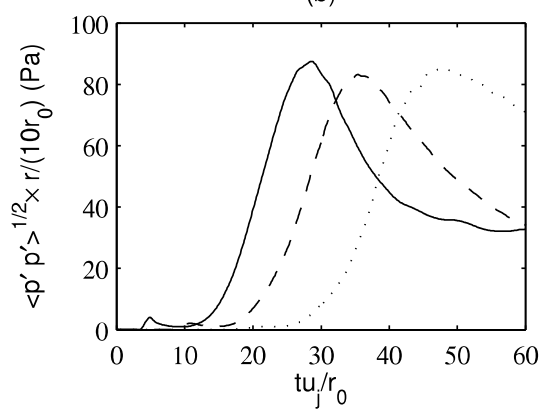

Figure 13. Time variations of the rms values of pressure fluctuations $p^{\prime}$ : (a) at $r=10 r_{0}$ for $\longrightarrow \operatorname{Re}_{D}=3,125$ and $\longrightarrow \operatorname{Re}_{D}=12,500$, (b) at $-r=5 r_{0},--r=10 r_{0}$ and $-\cdot-\cdot r=20 r_{0}$ for $\operatorname{Re}_{D}=3,125$ normalized using a $1 / r$ law.

3,125 and of $68 \mathrm{~Pa}$ at $t u_{j} / r_{0}=19$ for $\operatorname{Re}_{D}=12,500$. Given that $t_{c} u_{j} / r_{0}=21.6$ in the first case and $t_{c} u_{j} / r_{0}=16$ in the second case, the peak for $\operatorname{Re}_{D}=3,125$ may be related to the potential-core closing, but it cannot true for $\operatorname{Re}_{D}=12,500$. In this case, it most likely results from vortex pairings in the shear layers. For completeness, the rms pressure levels obtained for $\operatorname{Re}_{D}=3,125$ at the three radial positions $r=5 r_{0}, 10 r_{0}$ and $20 r_{0}$, normalized at $r=10 r_{0}$ using the $1 / r$ law, are represented in figure $13(\mathrm{~b})$. Peak values of 88,83 and $85 \mathrm{~Pa}$ are noted at $t u_{j} / r_{0}=28.6,35.4$ and 48 , respectively. The similarity in amplitude supports that the spreading of the sound waves radiated by the jet is spherical. Moreover, the time delays between the peaks indicate a radiation angle of roughly 45 degrees with respect to the axial direction.

\subsubsection{Pressure spectra}

The spectra of pressure fluctuations computed at $r=10 r_{0}$ at $t u_{j} / r_{0}=35,45$ and 55 for $\operatorname{Re}_{D}=3,125$ and at $t u_{j} / r_{0}=30,40$ and 50 for $\operatorname{Re}_{D}=12,500$ are represented in figure 14 as a function of axial wavenumber $k_{z} r_{0}$. At those times, the low-frequency acoustic waves generated around $t=t_{c}$ propagate at the radial position considered, as shown in figures 2 and 3. The three spectra for $\operatorname{Re}_{D}=3,125$ in figure 14(a) display very similar shapes. They contain strong components centered around a wavenumber of $k_{z} r_{0} \simeq 0.4$, and sharply decrease for $k_{z} r_{0}>1.6$. These components can be related to the presence of sound waves of wavelength of $\lambda_{z} \simeq 15 r_{0}$ in the pressure fields of figure 2. In the same way, the shape of the spectra for $\operatorname{Re}_{D}=12,500$ in figure 14 (b) does not depend much on the time considered. With respect to the spectra for the jet at $\operatorname{Re}_{D}=3,125$, they are comparable for $k_{z} r_{0}<1$, but show additional high-frequency components typically between $k_{z} r_{0}=1.6$ and 6.4 . Therefore, two contributions seem to 
(a)

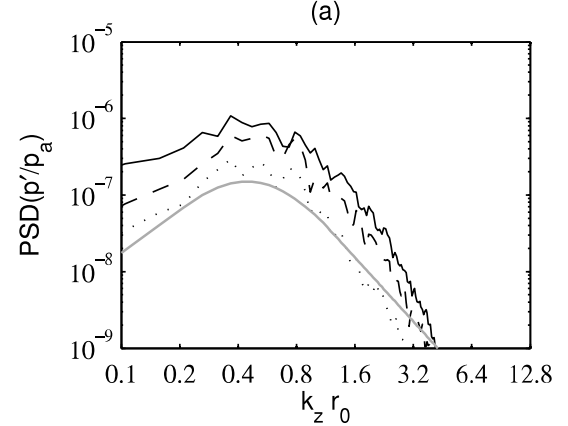

(b)

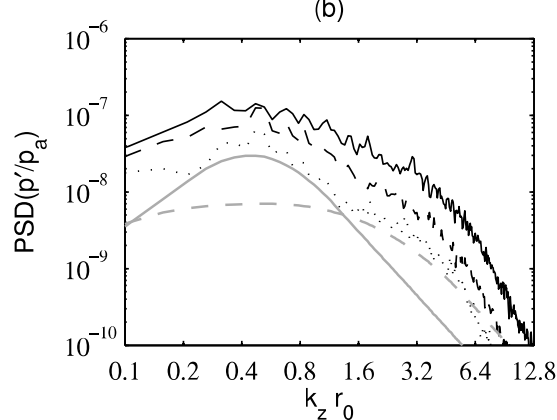

FiguRE 14. Representation of the power spectral densities of pressure fluctuations $p^{\prime} / p_{a}$ as a function of wavenumber $k_{z} r_{0}$, obtained at $r=10 r_{0}$ : (a) for $\operatorname{Re}_{D}=3,125$ at $-t u_{j} / r_{0}=35$, $---t u_{j} / r_{0}=45$ and $-\cdot-t u_{j} / r_{0}=55$, (b) for $\operatorname{Re}_{D}=12,500$ at $-t u_{j} / r_{0}=30$, $---t u_{j} / r_{0}=40$ and $-\cdot-t u_{j} / r_{0}=50 ;-$ large-scale and -- fine-scale similarity spectra of Tam et al. (1996)

emerge in the present sound spectra: a low-frequency contribution, noticeable at both Reynolds numbers, and a higher frequency one, disappearing when the Reynolds number is reduced down to $\operatorname{Re}_{D}=3,125$. This observation is consistent with those made based on the spectra of spatially-developing jets, used as evidence for the presence of two jet noise components (Tam 1998; Tam et al. 2008; Bogey \& Bailly 2006). It is also supported by the comparison of the present spectra with the large-scale and fine-scale similarity spectra found empirically by Tam et al. $(1996,2008)$ to fit the far-field spectra measured, respectively, in the downstream direction and in the upstream and sideline directions of both high subsonic and supersonic jets. Indeed, the spectra for $\operatorname{Re}_{D}=3,125$ do not differ much from the large-scale similarity spectrum when the peak of the latter is placed at $\left(k_{z} r_{0}\right)_{\text {peak }}=0.44$, whereas the spectra for $\operatorname{Re}_{D}=12,500$ visibly coincide with a combination of the two similarity spectra with $\left(k_{z} r_{0}\right)_{\text {peak }}=0.44$ and 0.55 .

The spectra of pressure fluctuations obtained at $r=20 r_{0}$ at $t u_{j} / r_{0}=45,55$ and 65 for $\operatorname{Re}_{D}=3,125$ and at $t u_{j} / r_{0}=40,50$ and 60 for $\operatorname{Re}_{D}=12,500$ are plotted in figure 15 as a function of the azimuthal mode. In all cases, the energy is maximum for the axisymmetric mode, decreases for higher modes, and is small or negligible for modes $n_{\theta}>4$. This azimuthal distribution of the acoustic pressure is close to that measured at low polar angles for spatially-developing subsonic jets (Juvé et al. 1979). For the present jets, in addition, the predominance of the axisymmetric mode is more marked as time passes, such as when lower axial angles are considered in the far field of spatial jets. That predominance is one important feature associated with the downstream subsonic jet noise component (Cavalieri et al. 2012).

\subsubsection{Pressure autocorrelations}

In the jet near field, the two-dimensional spatial autocorrelations of pressure fluctuations at radial position $r$ and time $t$ are calculated as

$$
\mathcal{R}_{p^{\prime} p^{\prime}}(\delta r, \delta z)=\frac{\left\langle p^{\prime}(r, \theta, z, t) p^{\prime}(r+\delta r, \theta, z+\delta z, t)\right\rangle}{\left\langle p^{\prime 2}(r, \theta, z, t)\right\rangle^{1 / 2}\left\langle p^{\prime 2}(r+\delta r, \theta, z+\delta z, t)\right\rangle^{1 / 2}}
$$

where $\delta r$ and $\delta z$ are the spatial separations in the radial and axial directions.

The correlation maps obtained at $r=10 r_{0}$ at $t u_{j} / r_{0}=35$ and 55 for $\operatorname{Re}_{D}=3,125$ and at $t u_{j} / r_{0}=30$ and 50 for $\operatorname{Re}_{D}=12,500$ are shown in figure 16. For the two jets, the orientation of the correlation spot changes with time, indicating a direction of 
(a)

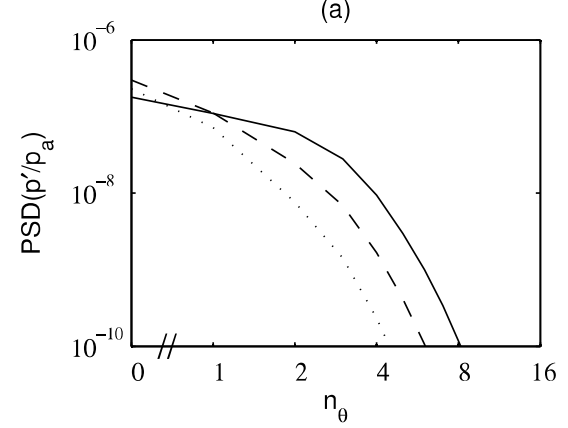

(b)

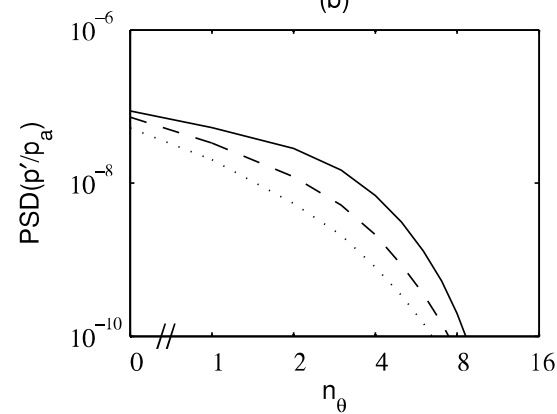

FIGURE 15. Representation of the power spectral densities of pressure fluctuations $p^{\prime} / p_{a}$ as a function of azimuthal mode $n_{\theta}$, obtained at $r=20 r_{0}$ : (a) for $\operatorname{Re}_{D}=3,125$ at $t u_{j} / r_{0}=45,---t u_{j} / r_{0}=55$ and $-\cdots t u_{j} / r_{0}=65$, (b) for $\operatorname{Re}_{D}=12,500$ at $t u_{j} / r_{0}=40,--t u_{j} / r_{0}=50$ and $-\cdots \cdot t u_{j} / r_{0}=60$.

(a)

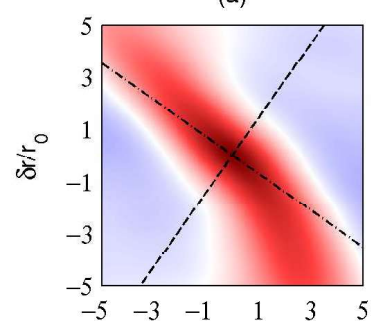

(c)

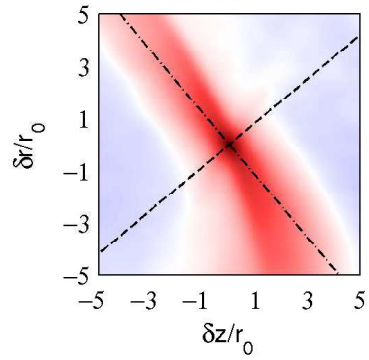

(b)

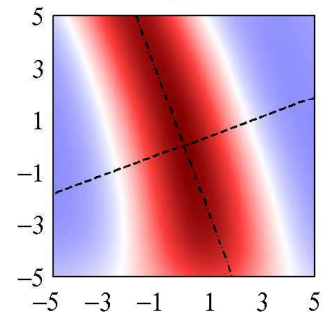

(d)

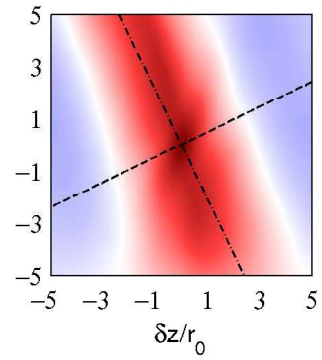

Figure 16. (Colour available at journals . cambridge.org/flm) Representation of the 2-D spatial correlations of pressure fluctuations obtained at $r=10 r_{0}$ for $\operatorname{Re}_{D}=3,125$ at (a) $t u_{j} / r_{0}=35$ and (b) $t u_{j} / r_{0}=55$, and for $\operatorname{Re}_{D}=12,500$ at (c) $t u_{j} / r_{0}=30$ and (d) $t u_{j} / r_{0}=50$; lines - - aligned with and - - - perpendicular to the average direction of acoustic propagation. The color scale ranges from -1 to 1 , from blue to red.

propagation getting closer to the jet direction. Its spatial extend also becomes larger. This is true both along the wave front, where correlation is strong over a very large distance, and normally to the wave front. For $\operatorname{Re}_{D}=12,500$, the correlation levels are lower than for $\operatorname{Re}_{D}=3,125$ for large separation distances, which is consistent with the richer frequency content of the pressure spectra reported above. Furthermore, weak additional correlation spots aligned in a direction opposite to that of the main spots are visible in figures $16(\mathrm{c}-\mathrm{d})$. They result from the acoustic waves propagating upstream in the jet near field, such as those noted in the bottom picture of figure 3 .

The radiation angles $\phi$ and the integral length scales $L_{\phi}$ computed along the average direction of propagation found from the spatial correlations of pressure at $r=10 r_{0}$ 
(a)

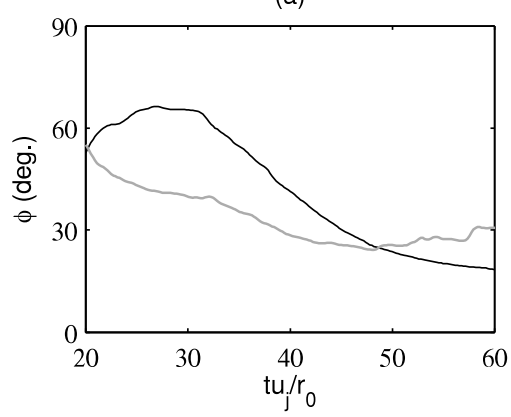

(b)

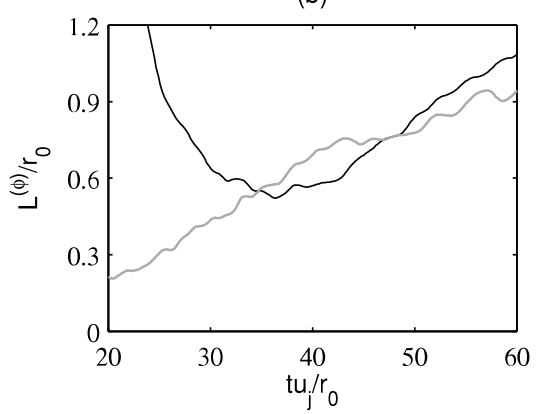

Figure 17. Time variations of (a) the radiation angle $\phi$ and (b) the integral length scale $L_{\phi} / r_{0}$ along the average direction of acoustic propagation at $r=10 r_{0}$ for $-\operatorname{Re}_{D}=3,125$ and $\operatorname{Re}_{D}=12,500$

are represented in figure 17. From $t u_{j} / r_{0} \simeq 30$ for $\operatorname{Re}_{D}=3,125$ and $t u_{j} / r_{0} \simeq 20$ for $\operatorname{Re}_{D}=12,500$ onwards, that is significantly after the jet potential-core closing, the radiation angles decrease with time in figure $17(\mathrm{a})$, from $\phi \simeq 60^{\circ}$ down to $\phi \simeq 30^{\circ}$ at $t u_{j} / r_{0}=50$. During the same period, the integral length scales grow and reach values of about $0.9 r_{0}$ at $t u_{j} / r_{0}=60$. The first result is expected given that, by construction, the waves remaining at $r=10 r_{0}$ long after their emission times can only be those propagating at low axial angles. The second one suggests an increase of the wavelength. This wavelength increase as the radiation angle decreases is consistent with the trend observed for the downstream noise component of spatial jets (Tam et al. 2008). It can also be explained by a wave packet modelling of the jet noise source, as illustrated by Papamoschou (2018), for instance.

\subsection{Flow-noise cross-correlations}

In order to identify links between the flow and sound fields of the jets, it is interesting to compute cross-correlations between flow quantities and acoustic pressure, as was done in several recent experimental and numerical investigations for spatially-developing jets (Seiner 1974; Schaffar 1979; Panda et al. 2005; Panda 2007; Bogey \& Bailly 2007; Grizzi \& Camussi 2012). In the present work, the axial velocity fluctuations $u_{z}^{\prime}$, the product $u_{z}^{\prime} u_{z}^{\prime}$ and the vorticity fluctuations $|\omega|^{\prime}$ obtained at $r=0$ and $r=r_{0}$, are correlated with the pressure fluctuations $p^{\prime}$ at $r=10 r_{0}$. These flow quantities are considered because they have been found in the previous studies mentioned above to correlate with far-field jet noise. Moreover, they appear in the source terms of acoustical analogies, namely $u_{z}^{\prime}$ and $u_{z}^{\prime} u_{z}^{\prime}$ in the shear-noise and self-noise source terms of Lighthill's analogy (Ribner 1964), and vorticity in the vortex sound theory (Powell 1964). The correlations are denoted by $\mathcal{C}_{u_{z}^{\prime} p^{\prime}}, \mathcal{C}_{u_{z}^{\prime} u_{z}^{\prime} p^{\prime}}$ and $\mathcal{C}_{|\omega|^{\prime} p^{\prime}}$. For $u_{z}^{\prime}$, for example, they are given by

$$
\mathcal{C}_{u_{z}^{\prime} p^{\prime}}\left(\delta z, t_{1}\right)=\frac{\left\langle u_{z}^{\prime}\left(r_{1}, \theta, z+\delta z, t_{1}\right) p^{\prime}\left(r_{2}, \theta, z, t_{2}\right)\right\rangle}{\left\langle u_{z}^{\prime 2}\left(r_{1}, \theta, z+\delta z, t_{1}\right)\right\rangle^{1 / 2}\left\langle p^{\prime 2}\left(r_{2}, \theta, z, t_{2}\right)\right\rangle^{1 / 2}}
$$

where $\delta z$ is the separation distance in the axial direction, $r_{1}=0$ or $r_{1}=r_{0}$, and $r_{2}=10 r_{0}$. In simple terms, the flow quantities at position $\left(r=r_{1}, z+\delta z\right)$ at time $t=t_{1}$ are correlated with the pressure fluctuations at position $\left(r=10 r_{0}, z\right)$ at $t=t_{2}$.

The correlations computed between the flow quantities at $r=0$ and pressure at $t_{2} u_{j} / r_{0}=40$ for $\operatorname{Re}_{D}=3,125$ and at $t_{2} u_{j} / r_{0}=35$ for $\operatorname{Re}_{D}=12,500$ are represented in figure 18 a functions of $\delta z$ and $t_{1}$. The velocity-pressure correlations obtained between $t_{2} u_{j} / r_{0}=30$ and 50 for $\operatorname{Re}_{D}=3,125$ and between $t_{2} u_{j} / r_{0}=30$ and 45 for 
(a)

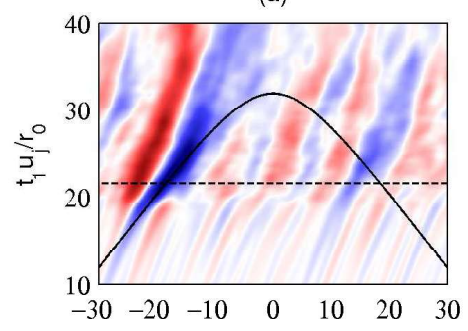

(d)

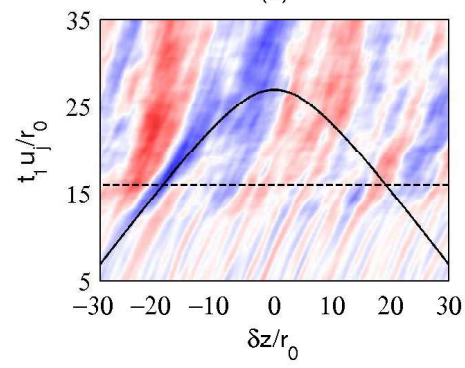

(b)

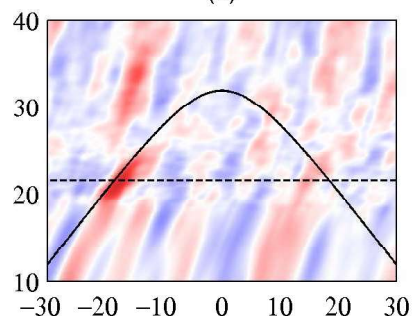

(e)

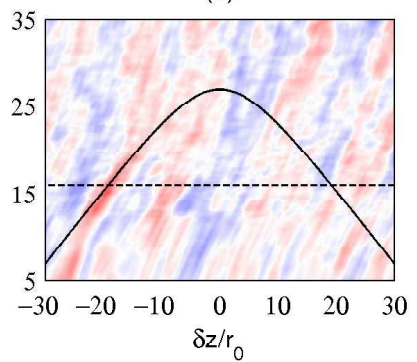

(c)

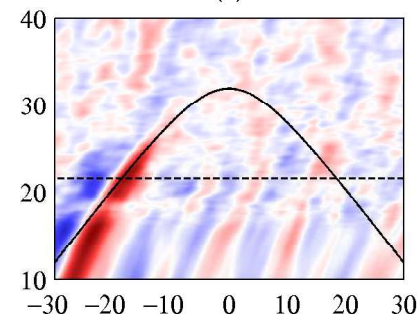

(f)

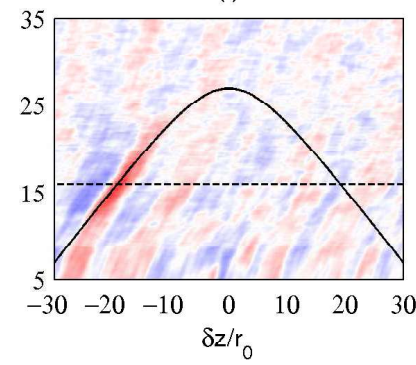

FIgURE 18. (Colour and movies online available at journals.cambridge.org/flm) Space-time correlations obtained between centerline quantities at $t=t_{1}$ and pressure fluctuations at $r=10 r_{0}$ (top) at $t_{2} u_{j} / r_{0}=40$ for $\operatorname{Re}_{D}=3,125$ and (bottom) at $t_{2} u_{j} / r_{0}=35$ for $\operatorname{Re}_{D}=12,500$ : $(\mathrm{a}, \mathrm{d}) \mathcal{C}_{u_{z}^{\prime} p^{\prime}},(\mathrm{b}, \mathrm{e}) \mathcal{C}_{u_{z}^{\prime} u_{z}^{\prime} p^{\prime}}$ and $(\mathrm{c}, \mathrm{f}) \mathcal{C}_{|\omega|^{\prime} p^{\prime}}$. The color scale ranges from -0.20 to 0.20 , from blue to red; - propagation at the ambient speed of sound; $--t_{1}=t_{c}$.

$\operatorname{Re}_{D}=12,500$ are displayed in the movies movie_fig18a and movie_fig $18 \mathrm{~b}$ available at journals.cambridge.org/flm. The dashed line represents the time of potential-core closing, and the solid line indicates a propagation at the ambient speed of sound between the centerline and near-field points. High levels of correlations, close to or higher than 0.2 for $\operatorname{Re}_{D}=3,125$ and 0.1 for $\operatorname{Re}_{D}=12,500$, are found near the intersection of the two lines for negative separation distances $\delta z$. This supports the presence of a sound source on the axis of both jets, radiating in the downstream direction, when the potential core disappears. The correlations are negative for $u_{z}^{\prime}$ and positive for $u_{z}^{\prime} u_{z}^{\prime}$ and $|\omega|^{\prime}$. They can be related to the intermittent arrival of low-velocity vortical structures in the jet core. Strong correlations also appear before the time of potential-core closing for vorticity, and afterward for $u_{z}^{\prime}$ and $u_{z}^{\prime} u_{z}^{\prime}$. They are due to the convection of the flow fluctuations in the jets, which will be illustrated later. These results are remarkably similar to those reported in Bogey \& Bailly (2007) for Mach number 0.9, spatially-developing jets at low and high Reynolds numbers.

For comparison, the correlations obtained between the flow quantities at $r=r_{0}$ and pressure at $r=10 r_{0}$ are represented in figure 19 in the same way as in figure 18 . The correlation levels are significantly lower than those evaluated from the centerline fluctuations as in spatial jets (Bogey \& Bailly 2007). They do not exceed values of 0.07 for $\operatorname{Re}_{D}=3,125$ and 0.06 for $\operatorname{Re}_{D}=12,500$. They even fail to emerge from the background noise in most cases, except for $\mathcal{C}_{u_{z}^{\prime} p^{\prime}}$ in figure 19(a) where an elongated correlation pattern similar, albeit very attenuated, to that visible in figure 18(a) appears for negative spatial separation distances. These results suggest that the sound source radiating in the downstream direction is not located in the center of the mixing layers.

Coming back to the correlations computed from centerline flow quantities, the corre- 
(a)

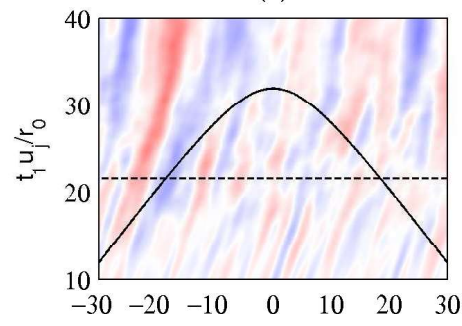

(d)

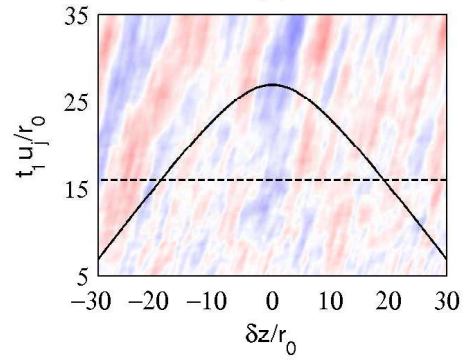

(b)

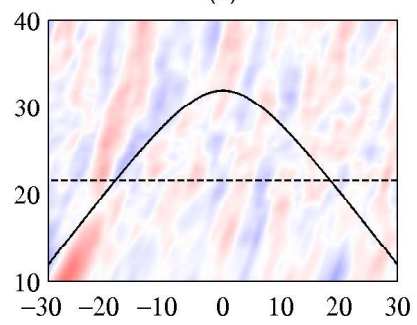

(e)

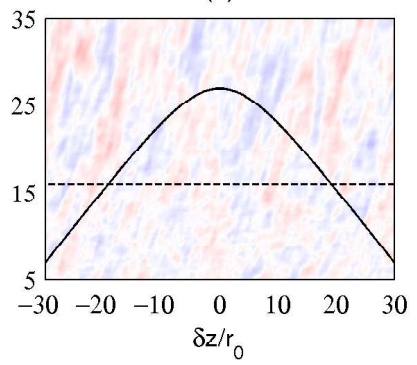

(c)

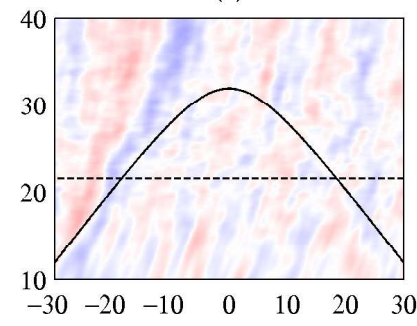

(f)

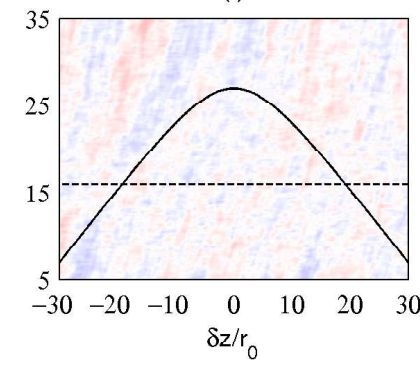

Figure 19. (Colour available at journals.cambridge.org/flm) Space-time correlations obtained between quantities at $r=r_{0}$ and $t=t_{1}$ and pressure fluctuations at $r=10 r_{0}$ (top) at $t_{2} u_{j} / r_{0}=40$ for $\operatorname{Re}_{D}=3,125$ and (bottom) at $t_{2} u_{j} / r_{0}=35$ for $\operatorname{Re}_{D}=12,500$ : (a,d) $\mathcal{C}_{u_{z}^{\prime} p^{\prime}}$, $(\mathrm{b}, \mathrm{e}) \mathcal{C}_{u_{z}^{\prime} u_{z}^{\prime} p^{\prime}}$ and $(\mathrm{c}, \mathrm{f}) \mathcal{C}_{|\omega|^{\prime} p^{\prime}}$. The color scale ranges from -0.20 to 0.20 , from blue to red; propagation at the ambient speed of sound; $--t_{1}=t_{c}$.

(a)

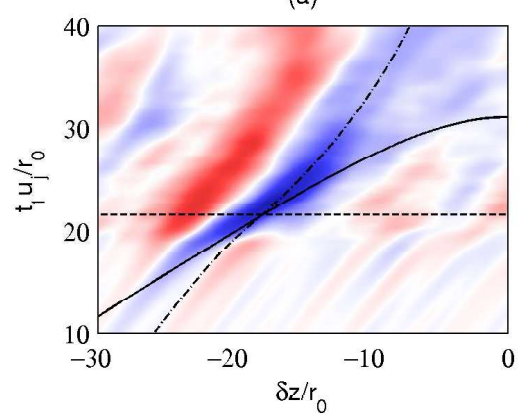

(b)

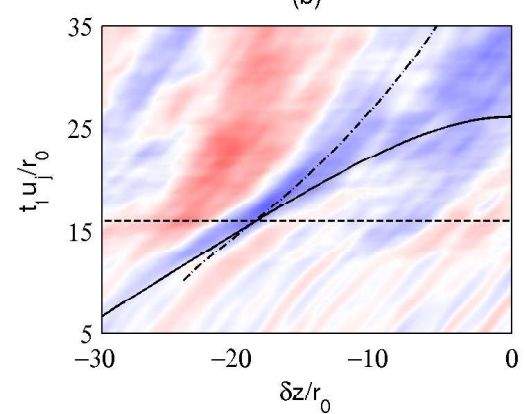

Figure 20. Space-time correlations $\mathcal{C}_{u_{z}^{\prime} p^{\prime}}$ obtained between velocity fluctuations at $r=0$ and $t=t_{1}$ and pressure fluctuations at $r=10 r_{0}$ (a) at $t_{2} u_{j} / r_{0}=40$ for $\operatorname{Re}_{D}=3,125$ and (b) at $t_{2} u_{j} / r_{0}=35$ for $\operatorname{Re}_{D}=12,500$. The color scale ranges from -0.30 to 0.30 , from blue to red; propagation at the ambient speed of sound; $---t_{1}=t_{c} ;-\cdots$ trajectory based on the convection velocity on the jet axis.

lations $\mathcal{C}_{u_{z}^{\prime} p^{\prime}}$ are presented again in figure 20, together with mixed lines built from the convection velocities on the jet axis given in figure 11(b). The correlation spots colored in blue turn out to follow these lines, revealing that their orientations result from the convection of the flow fluctuations in the jets (Bogey \& Bailly 2007). The time variations of these orientations are clearly seen in the corresponding movies.

In order to provide more quantitative results, the peak values of correlations determined from centerline fluctuations at time $t_{1}$ and pressure fluctuations at $t_{2} u_{j} / r_{0}=40$ for $\operatorname{Re}_{D}=3,125$ and $t_{2} u_{j} / r_{0}=35$ for $\operatorname{Re}_{D}=12,500$ are plotted in figure 21 as a 
(a)

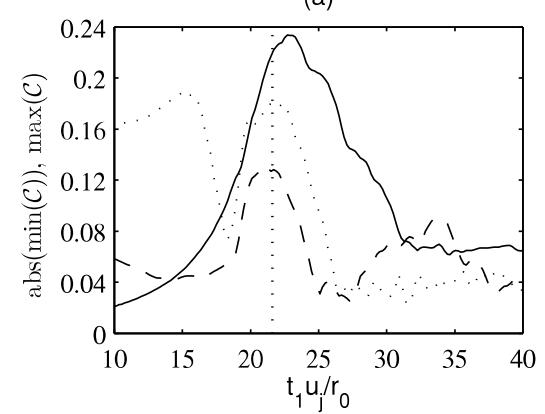

(b)

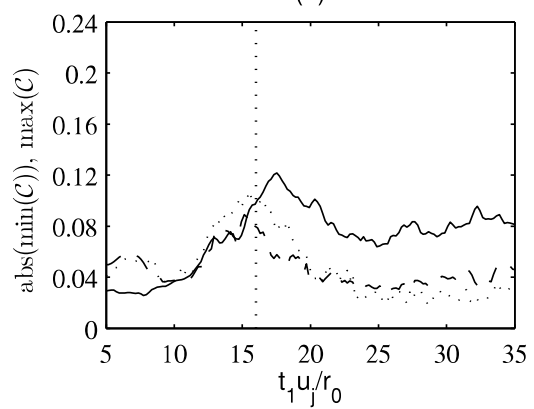

Figure 21. Representation of peak values of correlations between centerline quantities at $t=t_{1}$ and pressure fluctuations at $r=10 r_{0}$ (a) at $t_{2} u_{j} / r_{0}=40$ for $\operatorname{Re}_{D}=3,125$ and (b) at $t_{2} u_{j} / r_{0}=35$ for $\operatorname{Re}_{D}=12,500$ : $-\left|\min \left(\mathcal{C}_{u_{z}^{\prime} p^{\prime}}\right)\right|,---\max \left(\mathcal{C}_{u_{z}^{\prime} u_{z}^{\prime} p^{\prime}}\right),-\cdot-$ $\max \left(\mathcal{C}_{|\omega|^{\prime} p^{\prime}}\right)$, as a function of $t_{1} ; \cdots t_{1}=t_{c}$.

(a)

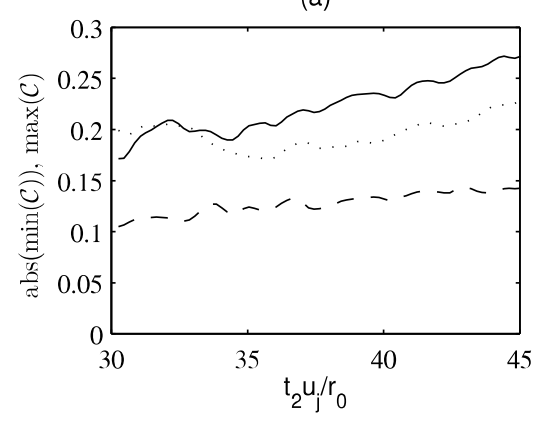

(b)

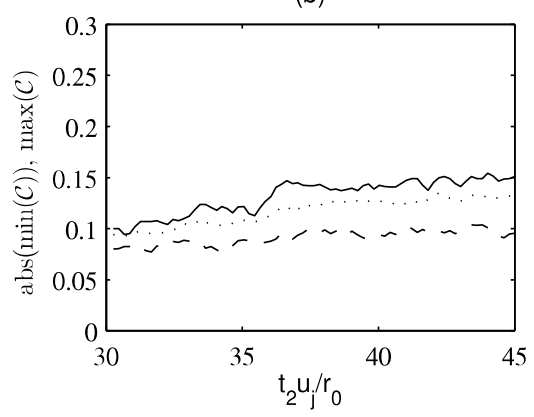

Figure 22. Representation of peak values of correlations between centerline quantities at $t=t_{1}$ and pressure fluctuations at $r=10 r_{0}$ at $t=t_{2}$ for (a) $\operatorname{Re}_{D}=3,125$ and (b) $\operatorname{Re}_{D}=12,500$ : $-\left|\min \left(\mathcal{C}_{u_{z}^{\prime} p^{\prime}}\right)\right|,---\max \left(\mathcal{C}_{u_{z}^{\prime} u_{z}^{\prime} p^{\prime}}\right),-\cdots \max \left(\mathcal{C}_{|\omega|^{\prime} p^{\prime}}\right)$, as a function of $t_{2}$.

function of $t_{1}$. Maximum values equal to $0.234,0.132$ and 0.180 for $\operatorname{Re}_{D}=3,125$ and to $1.122,0.890$ and 0.108 for $\operatorname{Re}_{D}=12,500$ are reached for $-\mathcal{C}_{u_{z}^{\prime} p^{\prime}}, \mathcal{C}_{u_{z}^{\prime} u_{z}^{\prime} p^{\prime}}$ and $\mathcal{C}_{|\omega|^{\prime} p^{\prime}}$, respectively, slightly after or at the time of potential-core closing. Significant levels are also obtained before $t=t_{c}$ for $\mathcal{C}_{|\omega|^{\prime} p^{\prime}}$ in figure 21(a). After the maximum values at $t \simeq t_{c}$, moreover, the levels decrease rather slowly for $-\mathcal{C}_{u_{z}^{\prime} p^{\prime}}$, supporting that the flow velocity feature correlating with the emitted sound waves persists long after the time of noise generation.

Finally, in the corresponding movies, the flow-noise correlation maps do not seem to depend much on the time of arrival of the acoustic waves at $r=10 r_{0}$. However, the correlation peak values gradually increase with time $t_{2}$, as highlighted in figure 22 . For $-\mathcal{C}_{u_{z}^{\prime} p^{\prime}}$, for instance, they grow from 0.17 up to 0.27 for $\operatorname{Re}_{D}=3,125$ and from 0.10 up to 0.15 for $\operatorname{Re}_{D}=12,500$ between $t_{2} u_{j} / r_{0}=30$ and 45 . Therefore, the flow-noise correlation levels are higher as the angle of radiation of the sound waves with respect to the flow direction is lower, which is in agreement with the experimental trends for spatial jets (Panda et al. 2005).

\subsection{Conditional averaging of the jet flow and pressure fields}

In order to visualize and better understand the noise generation mechanism related to the intermittent intrusion of turbulent structures in the core of the present jets, a conditional 
averaging method is applied in this section to the flow and acoustic fields provided by the simulations. The method consists in averaging a limited number of samples, selected using a trigger condition, in a signal or in a movie. It has been employed in the past in many studies to isolate a specific phenomenon from the background noise. For aeroacoustic purposes, it has enabled researchers, for example, to extract coherent structures and velocity events in mixing layers and jets (Dahan et al. 1978; Hileman et al. 2005; Camussi \& Grizzi 2014), and acoustic components in the pressure fields of free and impinging subsonic and supersonic jets (Kearney-Fisher et al. 2013; Akamine et al. 2016). Thanks to this method, Kastner et al. (2006), in particular, found that the rapid breakdown of the flow large-scale structures plays an important role in the generation of subsonic jet noise.

In the present work, the signal for the sample selection is the signal of axial velocity on the jet axis at the time $t_{c}$ of potential-core closing, and the trigger condition relies on the use of lower threshold. These choices are motivated by the fact that centerline axial velocity strongly correlates with the near-field pressure fluctuations long after $t_{c}$, and that it is negatively skewed, as shown in previous sections. The corresponding signals obtained in the first simulation runs of the two temporal jets are depicted in figure 23. For both Reynolds numbers, as expected from the profiles of skewness factors of velocity of figure $6(\mathrm{~b})$, intermittent negative spikes of velocity much lower than the jet velocity, even below $0.5 u_{j}$ in certain cases, can be seen. In the conditional averaging procedure, the trigger events are identified by detecting the minimum values of velocity below a threshold of $(2 / 3) u_{j}$, yielding a difference of approximately three standard deviations below the mean value for the signals considered. This threshold value is chosen arbitrarily, but the use of other velocity thresholds, such as $0.60 u_{j}$ and $0.75 u_{j}$, for example, does not change the results qualitatively but only quantitatively, as verified in appendix D. The trigger events separated by a distance of less than $10 r_{0}$ are discarded to avoid sample overlapping. This reduces for instance the number of samples to 5 in the two signals of figure 23, and leads to a total number equal to 45 for $\operatorname{Re}_{D}=3,125$ and 43 for $\operatorname{Re}_{D}=12,500$. The flow and pressure fields are then recorded over windows centered around the position $z_{c}$ of the trigger events, and the resulting snapshots are ensemble averaged. This is carried out at time $t_{c}$, but also at previous and subsequent times using the same synchronization with the negative spikes of axial centerline velocity at time $t_{c}$, in order to get access to the time evolution of the noise generation mechanism. The results obtained in the four azimuthal planes at $\theta=0, \pi / 2, \pi$ and $3 \pi / 2$ are averaged, which allows us to increase convergence, but also to focus on the generation of the axisymmetric sound waves dominating in the jet near field as shown by the spectra of figure 15. Despite the limited numbers of events selected, the averaged fields displayed in what follows clearly exhibit coherent features. This may be explained by the fact that these events are by far the most energetic ones in the flow.

The conditional fields of velocity fluctuations in the jet flow and of the pressure fluctuations outside, obtained at six times distributed every $5 r_{0} / u_{j}$ between $t_{c}-10 r_{0} / u_{j}$ and $t_{c}+15 r_{0} / u_{j}$, are represented in figure 24 for $\operatorname{Re}_{D}=3,125$ and in figure 26 for $\operatorname{Re}_{D}=12,500$. The corresponding movies movie_fig24 and movie_fig26 are provided at journals.cambridge.org $/ \mathrm{flm}$. The conditional fields of vorticity fluctuations at $t_{c}-$ $5 r_{0} / u_{j}, t_{c}$ and $t_{c}+5 r_{0} / u_{j}$ are also given in figures 25 and 27 . In the figures, velocity contours are drawn for the values of one third and two thirds of the mean centerline velocity in order to make the extent of the mixing layer visible. Similar features are observed for the two jets despite the difference in Reynolds number. The amplitudes are however lower in the pressure field for the higher Reynolds number case, in agreement with the results of section 3.3 . 
(a)

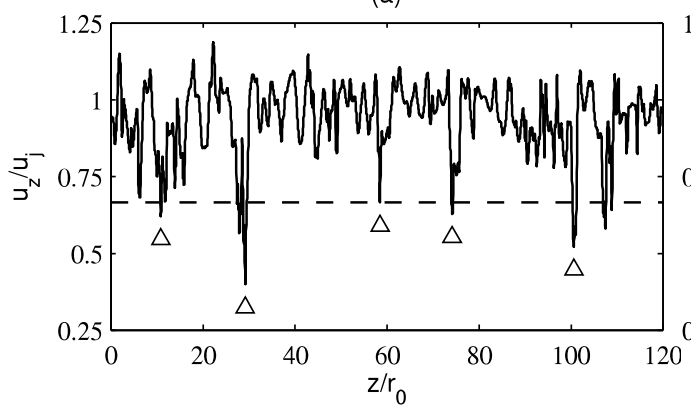

(b)

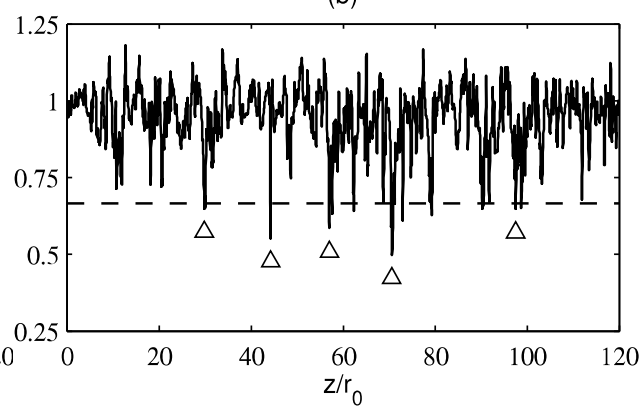

FIgURE 23. Axial velocity obtained at $r=0$ and $t=t_{c}$ for (a) $\operatorname{Re}_{D}=3,125$ and

(b) $\operatorname{Re}_{D}=12,500 ;---u_{z} / u_{j}=2 / 3 ; \triangle$ events selected for the conditional averaging.

(a)

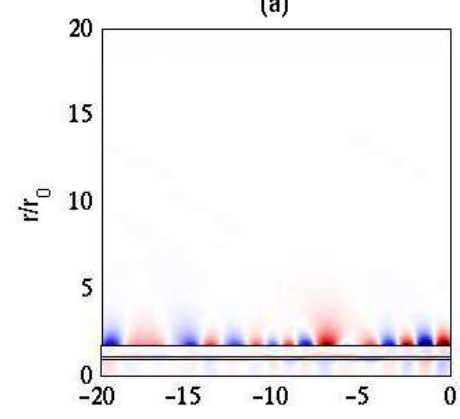

(d)

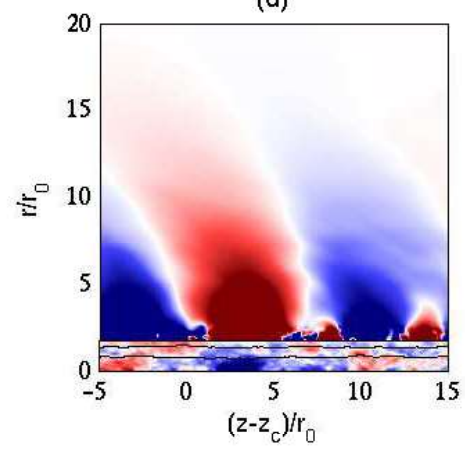

(b)

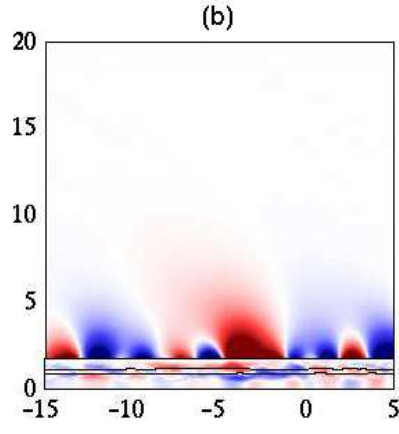

(e)

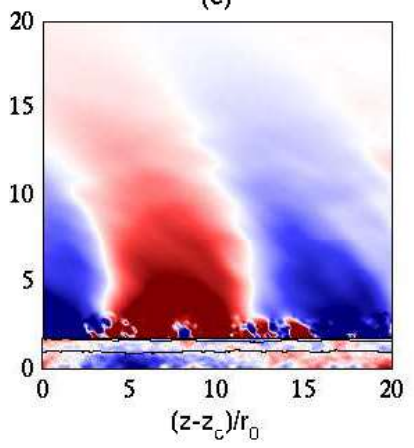

(c)

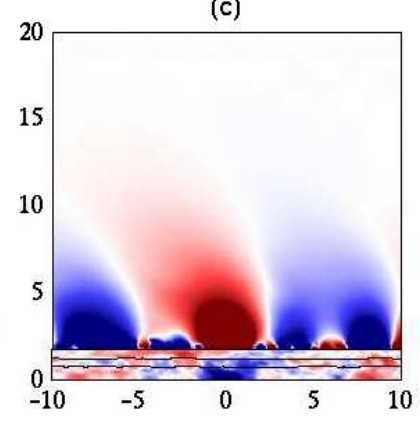

(f)

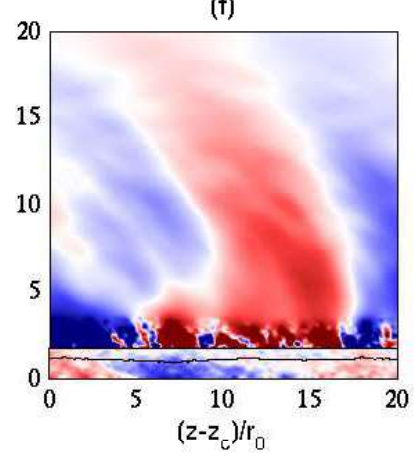

FigURE 24. (Colour and movie online available at journals cambridge.org/flm) Representation of the fluctuations of axial velocity for $r \leq 1.75 r_{0}$ and of pressure otherwise, obtained for $\operatorname{Re}_{D}=3,125$ at (a) $t_{c}-10 r_{0} / u_{j}$, (b) $t_{c}-5 r_{0} / u_{j}$, (c) $t_{c}$, (d) $t_{c}+5 r_{0} / u_{j}$, (e) $t_{c}+10 r_{0} / u_{j}$ and (f) $t_{c}+15 r_{0} / u_{j}$ using conditional averaging; $u_{z}=(1 / 3)\left\langle u_{z}(r=0)\right\rangle$ and $u_{z}=(2 / 3)\left\langle u_{z}(r=0)\right\rangle$. The color scales range from $-0.075 u_{j}$ up to $0.075 u_{j}$ and from $-90 \mathrm{~Pa}$ up to $90 \mathrm{~Pa}$, from blue to red.

At the time of potential-core closing, in figures 24(c) and 25(b) for $\operatorname{Re}_{D}=3,125$, and in figures $26(\mathrm{c})$ and 27 (b) for $\operatorname{Re}_{D}=12,500$, spots of strong negative and positive values are found, respectively, in the velocity and vorticity fields on the jet axis around $z=z_{c}$, as expected due to the trigger condition used in the averaging procedure. In the pressure fields, lobes of positive fluctuations located at $z \simeq z_{c}$ and surrounded by two smaller lobes of negative fluctuations are noticed. They can be regarded as the traces of the turbulent structures in the jet near field (Arndt et al. 1997). 
(a)

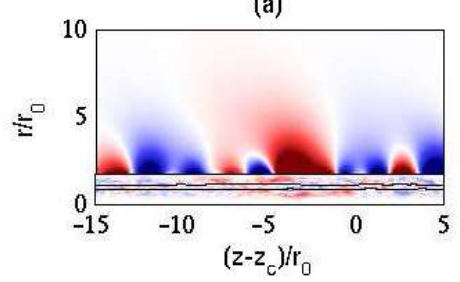

(b)

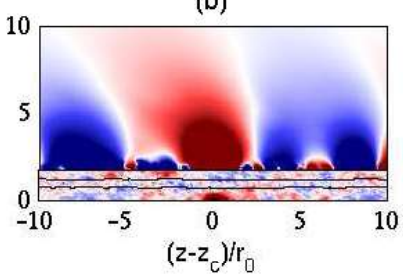

(c)

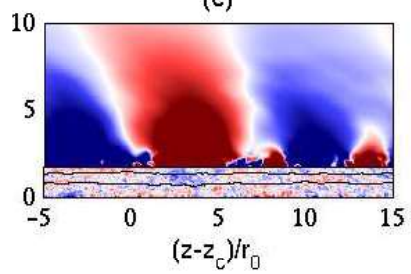

Figure 25. (Colour available at journals . cambridge.org/flm) Representation of the fluctuations of vorticity norm for $r \leq 1.75 r_{0}$ and of pressure otherwise, obtained for $\operatorname{Re}_{D}=3,125$ at (a) $t_{c}-5 r_{0} / u_{j}$, (b) $t_{c}$ and (c) $t_{c}+5 r_{0} / u_{j}$ using conditional averaging; $u_{z}=(1 / 3)\left\langle u_{z}(r=0)\right\rangle$ and $u_{z}=(2 / 3)\left\langle u_{z}(r=0)\right\rangle$. The color scales range from $-0.75 u_{j} / r_{0}$ up to $0.75 u_{j} / r_{0}$ and from $-90 \mathrm{~Pa}$ up to $90 \mathrm{~Pa}$, from blue to red.

(a)

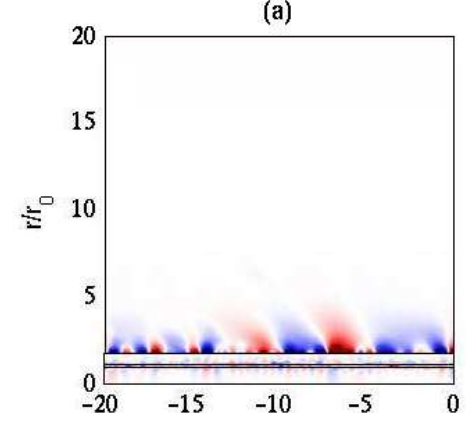

(d)

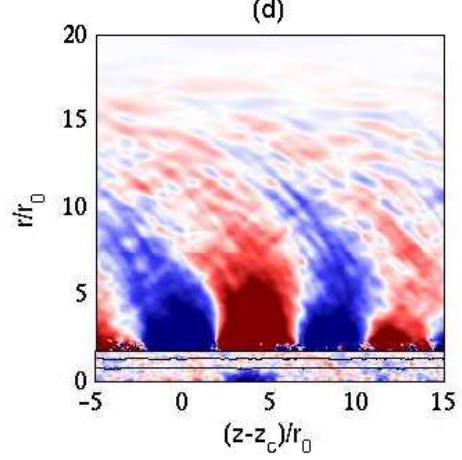

(b)

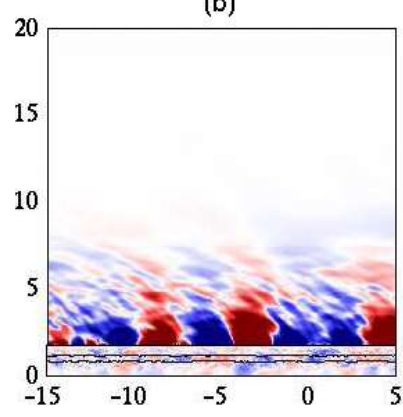

(e)

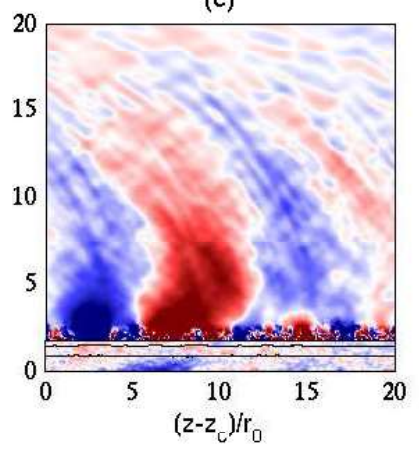

(c)

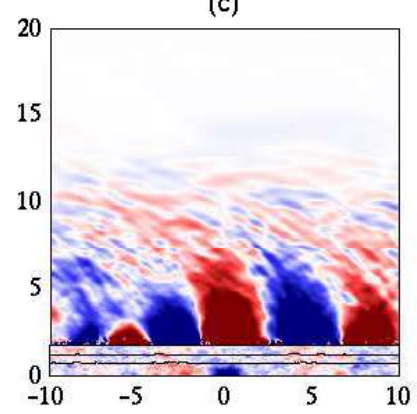

(f)

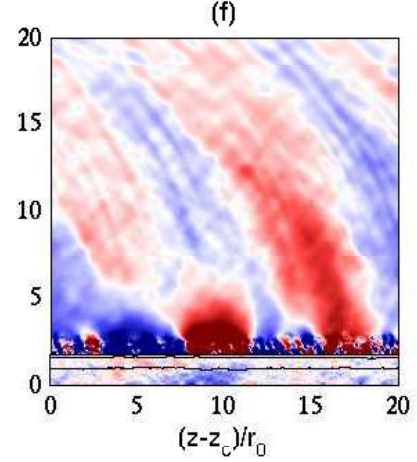

FIgURE 26. (Colour and movie online available at journals cambridge.org/flm) Representation of the fluctuations of axial velocity for $r \leq 1.75 r_{0}$ and of pressure otherwise, obtained for $\operatorname{Re}_{D}=12,500$ at (a) $t_{c}-10 r_{0} / u_{j}$, (b) $t_{c}-5 r_{0} / u_{j}$, (c) $t_{c}$, (d) $t_{c}+5 r_{0} / u_{j}$, (e) $t_{c}+10 r_{0} / u_{j}$ and (f) $t_{c}+15 r_{0} / u_{j}$ using conditional averaging; $u_{z}=(1 / 3)\left\langle u_{z}(r=0)\right\rangle$ and $u_{z}=(2 / 3)\left\langle u_{z}(r=0)\right\rangle$. The color scales range from $-0.075 u_{j}$ up to $0.075 u_{j}$ and from $-40 \mathrm{~Pa}$ up to $40 \mathrm{~Pa}$, from blue to red.

The axial profiles of velocity and vorticity fluctuations at $r=0$ and of pressure fluctuations at $r=2.5 r_{0}$ at $t=t_{c}$ are plotted in figure 28. For both jets, negative and positive spikes emerge for velocity and vorticity. They are centered around $z=z_{c}$, are of length $0.6 r_{0}$, and exhibit extremum values of $-0.38 u_{j}$ and $2.5 u_{j} / r_{0}$ for $\operatorname{Re}_{D}=3,125$ and of $-0.36 u_{j}$ and $3.5 u_{j} / r_{0}$ for $\operatorname{Re}_{D}=12,500$. At the same axial position, a hump of positive values peaking at $324 \mathrm{~Pa}$ for $\operatorname{Re}_{D}=3,125$ and at $215 \mathrm{~Pa}$ for $\operatorname{Re}_{D}=12,500$ is seen in the pressure profiles. It is the central part of the instability wave, of typical wavelength 
(a)

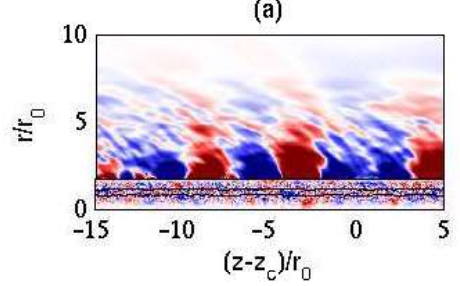

(b)

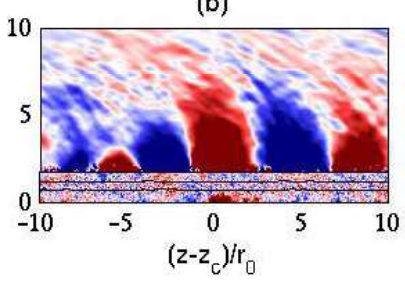

(c)

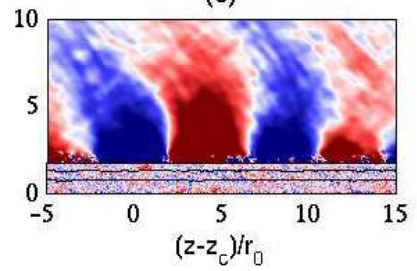

Figure 27. (Colour available at journals. cambridge.org/flm) Representation of the fluctuations of vorticity norm for $r \leq 1.75 r_{0}$ and of pressure otherwise, obtained for $\operatorname{Re}_{D}=12,500$ at (a) $t_{c}-5 r_{0} / u_{j}$, (b) $t_{c}$ and (c) $t_{c}+5 r_{0} / u_{j}$ using conditional averaging; $u_{z}=(1 / 3)\left\langle u_{z}(r=0)\right\rangle$ and $u_{z}=(2 / 3)\left\langle u_{z}(r=0)\right\rangle$. The color scales range from $-0.75 u_{j} / r_{0}$ up to $0.75 u_{j} / r_{0}$ and from $-40 \mathrm{~Pa}$ up to $40 \mathrm{~Pa}$, from blue to red.

(a)

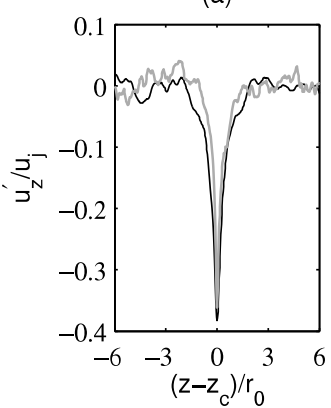

(b)

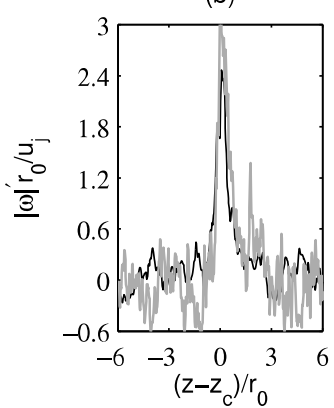

(c)

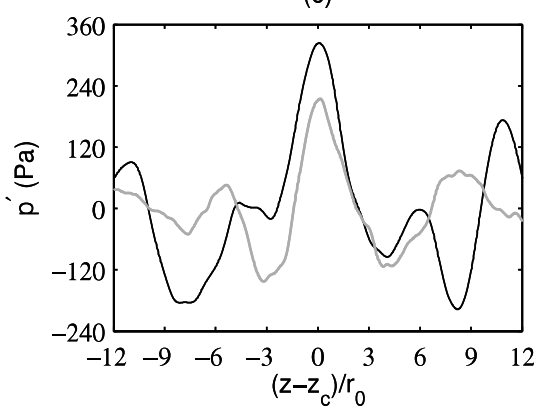

FIGURE 28. Representation of the fluctuations of (a) axial velocity and (b) vorticity norm at $r=0$, and of (c) pressure at $r=2.5 r_{0}$, obtained at $t=t_{c}$ using conditional averaging for $\longrightarrow \operatorname{Re}_{D}=3,125$ and $\longrightarrow \operatorname{Re}_{D}=12,500$.

$12 r_{0}$, appearing in the pressure fields of figures 24(c) and 26(c). Therefore, when the potential core closes, the eruption of shear-layer vortical structures through the centre of the jet results in a velocity deficit and a vorticity excess in the flow, and in hydrodynamic pressure waves in its immediate vicinity.

The evolutions of these flow and pressure patterns are revealed in figures 24-27 and in the corresponding movies. In both jets, before the potential-core closing, a spot of lower velocity and higher vorticity level relative to the background flow field develops in the interfacial region between the mixing layer and the potential core. It is discernible at a very early stage, at $t_{c}-10 r_{0} / u_{j}$ around $z=z_{c}-7 r_{0}$ in the velocity fields of figures 24 (a) and 26(a), and at $t_{c}-5 r_{0} / u_{j}$ around $z=z_{c}-3.5 r_{0}$ in the vorticity fields of figures 25(b) and $27(\mathrm{~b})$. Its strength is characterized in figure 29 showing the extremum values of velocity and vorticity fluctuations at $r=0$ and between $r=0$ and $r=(2 / 3) r_{0}$ as a function of time. In absolute values, they first increase relatively slowly, but sharply rise after $t_{c}-3 r_{0} / u_{j}$. They reach a peak at $t \simeq t_{c}$ when the vortical spot arrives on the jet axis, and thereafter collapse. For $t \geq t_{c}$, the structure extracted by the conditional averaging also decelerates. It is thus located consecutively around $z=z_{c}, z_{c}+4 r_{0}, z_{c}+7 r_{0}$ and $z_{c}+9 r_{0}$ in figures 26(c-f), for example. Moreover, the velocity deficit appears to persist over a long time period, and is still quite visible at $t_{c}+15 r_{0} / u_{j}$ for both jets, with a large extent in the axial direction. On the contrary, the vorticity excess vanishes rapidly, and is hardly detectable at $t_{c}+5 r_{0} / u_{j}$ in figures $25(\mathrm{c})$ and $27(\mathrm{c})$.

The development and disappearance of the high-vorticity spot on the high-velocity side of the mixing layer is accompanied by the growth and decay of a hydrodynamic pressure 
(a)

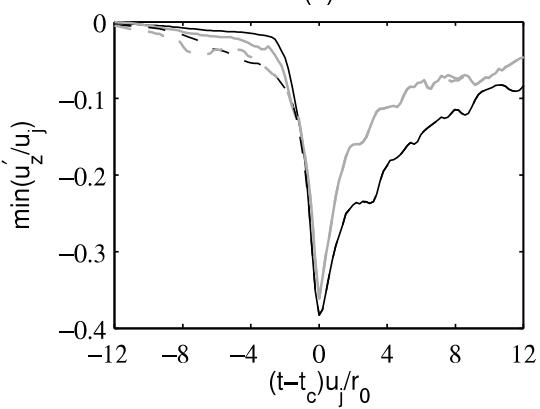

(b)

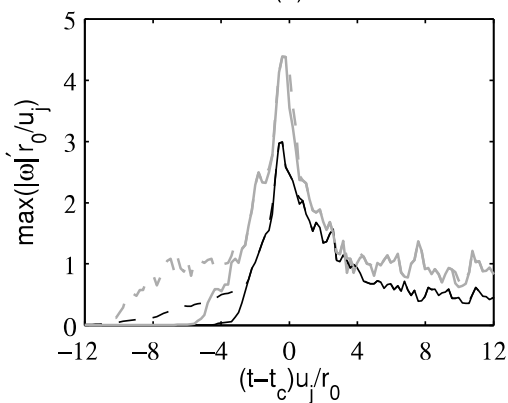

Figure 29. Time variations of (a) the minimum values of axial velocity fluctuations and (b) the maximum values of vorticity norm fluctuations obtained at $r=0$ for $-\operatorname{Re}_{D}=3,125$ and $-\operatorname{Re}_{D}=12,500$, and between $r=0$ and $r=(2 / 3) r_{0}$ for $--\operatorname{Re}_{D}=3,125$ and $---\operatorname{Re}_{D}=12,500$ using conditional averaging.

wave in the jet near field. To quantify this phenomenon, the normalized maximum values of pressure fluctuations obtained at $r=2.5 r_{0}$, as well as the corresponding values averaged over $2 r_{0} \leq r \leq 3 r_{0}$ are represented in figure 30(a). For both jets, the two profiles are very similar to each other, indicating that the shape of the signal envelope does not depend appreciably on the radial position. In addition, the peak values of pressure fluctuations are achieved at $t \simeq t_{c}+2 r_{0} / u_{j}$. Interestingly, this is roughly the time when the correlations between the centerline velocity and the radiated noise are the strongest, refer to figure 21, suggesting that noise generation occurs at that moment.

Outside the jets, the emission and propagation of acoustic waves in the downstream direction are then clearly visible for both jets. One dominant wave of positive pressure, and two weaker waves of negative pressure on both sides, are radiated. They are attached to the flow velocity deficit at $t=t_{c}$, but progressively move further away at later times as they travel at the speed of sound, more rapidly than the flow structures. The noise generation mechanism highlighted here strongly resembles that associated with a wave packet, exemplified in Tam et al. (2008), Suzuki (2010) and Jordan \& Colonius (2013). In the present work, it is clearly identified in a subsonic jet flow without resorting to any wave packet modelling.

The axial positions of the wave packets in the present jets, given in practice by those of the pressure peaks in the near field, are presented in figure 30(b) as a function of time. They are nearly identical for the two jets, regardless of the Reynolds number. Furthermore, they are very close to the positions of the minimum values of velocity in the flow, also depicted in the figure. This demonstrates the connection between the structure developing at the edge of the potential core and the hydrodynamic wave outside the jet flow. Both appear to travel at a rather uniform velocity between $t_{c}-10 r_{0} / u_{j}$ and $t_{c}+5 r_{0} / u_{j}$, but subsequently at a velocity decreasing with time.

The velocities estimated from the axial positions of the wave packets over segments of duration $5 u_{j} / r_{0}$ are shown in figure 31 . For both jets, despite the presence of oscillations, they are close to 65 per cent of the initial jet velocity at $t_{c}-10 r_{0} / u_{j}$, then gradually increase up to approximately $0.75 u_{j}$ and finally falls down for $t>t_{c}+5 r_{0} / u_{j}$. Compared to the convection velocities calculated at $r=0$ and $r=r_{0}$ from velocity cross-correlations, they differ significantly. First, they are always higher than the convection velocity at $r=r_{0}$, which can be related to the fact that the structure developing in the flow is not located in the center of the mixing layer, as mentioned above. Second, at $t \simeq t_{c}$, they are lower than the convection velocity at $r=0$ suggesting that in average, the structure 
(a)

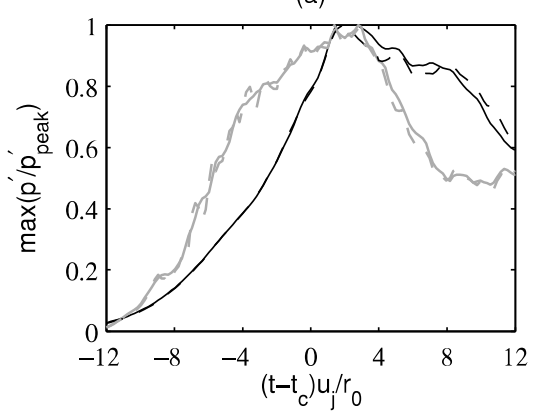

(b)

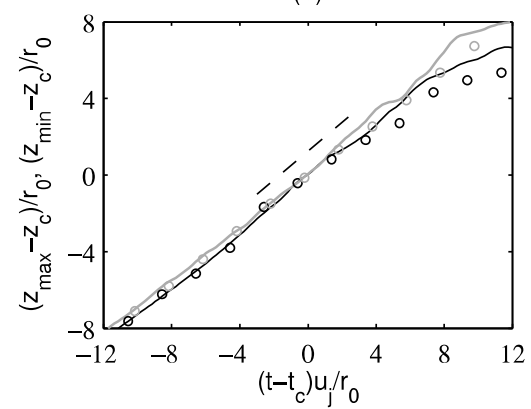

Figure 30. Time variations (a) of the maximum values of pressure fluctuations, normalized by their peak values, averaged over $2 r_{0} \leq r \leq 3 r_{0}$ for $-\operatorname{Re}_{D}=3,125$ and $\operatorname{Re}_{D}=12,500$ and at $r=2.5 r_{0}$ for $---\operatorname{Re}_{D}=3,125$ and $---\operatorname{Re}_{D}=12,500$, (b) of the axial positions of the pressure peaks averaged over $2 r_{0} \leq r \leq 3 r_{0}$ for $-\operatorname{Re}_{D}=3,125$ and $\longrightarrow \operatorname{Re}_{D}=12,500$ and of the minimum values of axial velocity between $r=0$ and $r=(2 / 3) r_{0}$ for $\circ \operatorname{Re}_{D}=3,125$ and $\circ \operatorname{Re}_{D}=12,500 ;---$ propagation at $0.8 u_{j}$.

(a)

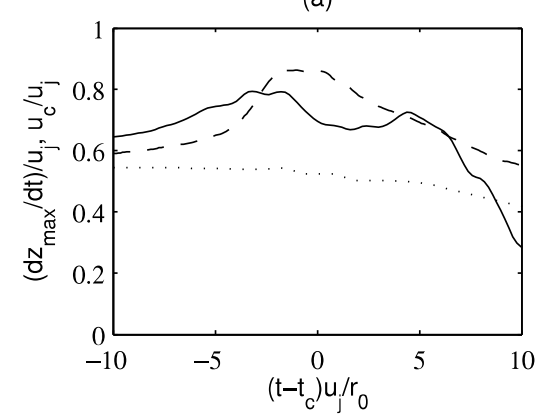

(b)

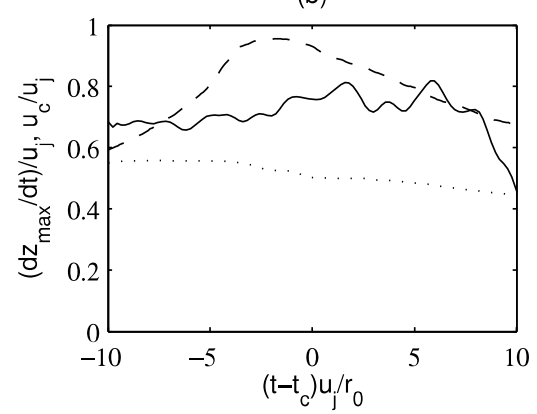

Figure 31. Time variations of

the velocities calculated from the positions of the near-field pressure peaks represented in figure 30(b) and of the convection velocities at - - $r=0$ and $-\cdots r=r_{0}$ for (a) $\operatorname{Re}_{D}=3,125$ and (b) $\operatorname{Re}_{D}=12,500$.

accelerates less than it could have been expected when the potential core closes. Inversely, it appears to slow down suddenly shortly after.

Finally, the present conditional fields provide explanations for the properties of the correlation maps obtained between flow and acoustic fluctuations for the temporal jets, but also for spatially-developing jets (Schaffar 1979; Bogey \& Bailly 2007). The absence of velocity deficit and vorticity excess in the mixing layer seems to be responsible for the negligible correlations computed from the flow signals at $r=r_{0}$. The persistence of a velocity deficit over a long period and the rapid disappearance of a vorticity excess on the jet axis may also be the reasons for which the correlations based on the centerline signals remain significant long after the time of sound generation for velocity but not for vorticity. Lastly, several pressure waves of alternating sign are emitted during the noise generation process, which may be the cause of the presence of the multiple spots of notable level in the correlation fields.

\section{Conclusion}

In this paper, noise generation in temporally-developing subsonic round jets is investigated. For that purpose, the flow and the near pressure fields computed for isothermal jets 
at a Mach number of 0.9 and Reynolds numbers of 3,125 and 12,500 using direct numerical simulation are detailed. Cross-correlations between the two fields are also calculated in order to localize possible sound sources in the jets. Overall, despite the difference in Reynolds number, leading to a more rapid flow development and more high-frequency components for the higher-Reynolds-number jet, the results for the two jets are very similar. In both cases, when the jet potential core closes, shear-layer turbulent structures intermittently intrude inside the jet, and high-amplitude low-frequency waves are radiated in the downstream direction. These waves are significantly correlated with the axial velocity and vorticity fluctuations on the jet centerline. These results suggest that the mechanism responsible for the downstream noise component of spatially-developing subsonic jets is also encountered in temporal jets. Therefore, it appears not to depend on the presence of a potential core of finite length, of a nozzle, or on the streamwise spreading of the jet flow, but to be linked to the physics of the interactions and merging of parallel mixing layers.

In the present jets, that mechanism is extracted by a procedure of conditional averaging of the flow and sound fields using a sample synchronization with the minimum values of centerline velocity at the time of potential-core closing $t_{c}$. It occurs over a long time period, and evolves large-scale coherent features of the flow. In summary, a spot of lower velocity and higher vorticity level relative to the background flow field develops at the inner edge of the mixing layer, reaches a peak intensity on the jet axis at $t_{c}$, and then disappears. Simultaneously, a hydrodynamic wave packet grows and decays in the near pressure field. Both travel at a velocity initially slightly increasing with time, but rapidly decreasing shortly after $t_{c}$. In the acoustic field, sound waves are emitted in the flow direction from the wave packet. Further studies will be based on extra post-processings of the present simulation data. Additional simulations of temporally-developing jets, at different Mach numbers for instance, will also be performed in order to get more information about the noise generation mechanism revealed in this work. Finally, it will be necessary to go back and consider spatial jets to determine whether that mechanism can be isolated in such flows.

The present work was granted access to the High-Performance-Computing resources of FLMSN (Fédération Lyonnaise de Modélisation et Sciences Numériques), partner of EQUIPEX EQUIP@MESO, and of IDRIS (Institut du Développement et des Ressources en Informatique Scientifique) under the allocation 2016-2a0204 made by GENCI (Grand Equipement National de Calcul Intensif). It was performed within the framework of the Labex CeLyA of Université de Lyon, operated by the French National Research Agency (Grant No. ANR-10-LABX-0060/ANR-11-IDEX-0007).

\section{Appendix A}

In this first appendix, the extra simulation of a temporal jet at $\operatorname{Re}_{D}=50,000$ is reported. The jet flow is initially set up and forced as described in section 2.1 for the two jets at lower Reynolds numbers considered in this study. Following the variations of $\delta_{\theta} / r_{0}$ with the Reynolds number, in particular, the momentum thickness of the mixing layer is equal to $\delta_{\theta}=0.0089 r_{0}$ at $t=0$, which is twice as thin as for the jet at $\operatorname{Re}_{D}=12,500$. In the simulation, the grid is finer compared to that defined in section 2.3 for the two other jets to keep a very high resolution. It contains $n_{r} \times n_{\theta} \times n_{z}=657 \times 1024 \times 3,200=2.2$ billion points, and is characterized by mesh spacings of $\Delta r=0.003 r_{0}$ at $r=r_{0}$ and $\Delta r=0.0125 r_{0}$ on the jet axis, and of $\Delta z=0.0125 r_{0}$. Therefore, it extends only up to $z=40 r_{0}$ in the axial direction. A total number 18,600 iterations are performed, for a 

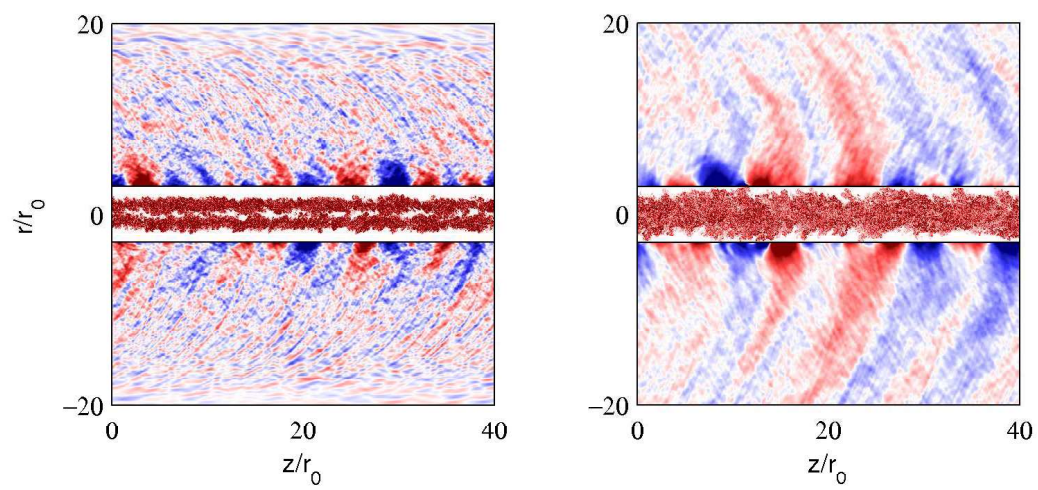

FIgURE 32. (Colour and movie online available at journals.cambridge.org/flm) Representation of vorticity norm for $r \leq 3 r_{0}$ and of pressure fluctuations otherwise, obtained for $\operatorname{Re}_{D}=50,000$ at $t u_{j} / r_{0}=20$ and 45 , from left to right. The color scales range up to the level of $5 u_{j} / r_{0}$ for vorticity, and from $-200 \mathrm{~Pa}$ to $200 \mathrm{~Pa}$ for pressure, from blue to red.

final time of $t=50 r_{0} / u_{j}$. Only one run is carried out, preventing the presentation of statistical results.

Snapshots of the vorticity norm and pressure fluctuations obtained in the $(z, r)$ plane at $t u_{j} / r_{0}=20$ and 45 , that is just before and well after the time of potential-core closing, are however shown in figure 32 and in the movie movie_fig32 available at journals .cambridge.org/flm. Large-scale hydrodynamic waves and acoustic waves of short wavelength are found in the pressure field at both times. Above all, low-frequency sound waves are seen to propagate in the downstream direction at the later time. They strongly resemble and have amplitudes similar to those obtained for the jet at $\operatorname{Re}_{D}=12,500$ in figure 3. These results suggest that the downstream noise component obtained for the temporal jets at $\operatorname{Re}_{D}=3,125$ and $\operatorname{Re}_{D}=12,500$ persists at higher Reynolds numbers. This component also seems not to depend significantly on the shear-layer initial momentum thickness.

\section{Appendix B}

In this second appendix, the space correlations of axial velocity fluctuations obtained at $r=0$ and $r=r_{0}$ in the the two temporal jets at $\operatorname{Re}_{D}=3,125$ and $\operatorname{Re}_{D}=12,500$ at times $t r_{0} / u_{j}=10,20,30$ and 40 are represented in figure 33 as a function of separation distance $\delta z$ up to $\delta z=60 r_{0}$, which is half the axial extent of the computational domain, in order to examine the influence of the periodic conditions, and to detect a possible bias due to these conditions. For separation distances $\delta z>5 r_{0}$, the correlation levels at $r=0$ in figures $33(\mathrm{a}, \mathrm{c})$ are small, typically below 0.05 , and decrease with time as the jets develop. The levels at $r=r_{0}$ in figures 33(b,d) are much smaller, and even negligible, due to a better convergence thanks to the averaging in the azimuthal direction. In all cases, in particular, no correlation hump emerges around $\delta z=60 r_{0}, 30 r_{0}$ and $15 r_{0}$, corresponding to one half, one quarter and one eighth of the domain size. Therefore, the periodic conditions in the axial direction do not appear to distort the jet flow development.

\section{Appendix $\mathbf{C}$}

In this third appendix, the time variations of mean axial velocity on the jet centerline, of peak axial turbulence intensity and of the rms values of pressure fluctuations at $r=10 r_{0}$, 
(a)

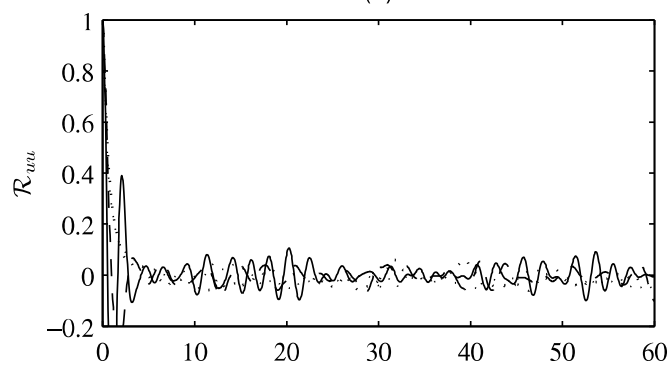

(c)

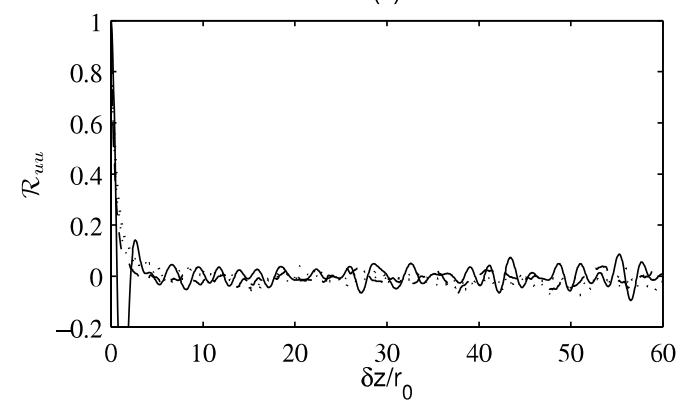

(b)

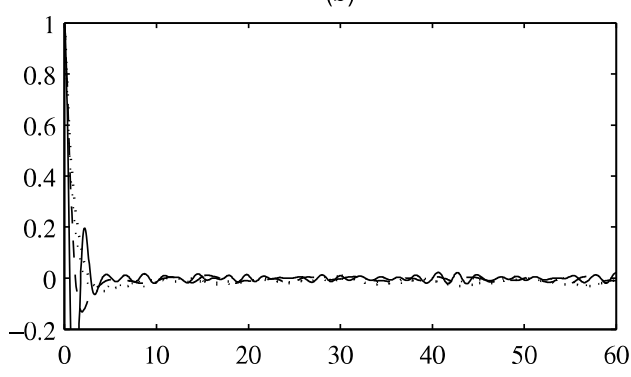

(d)

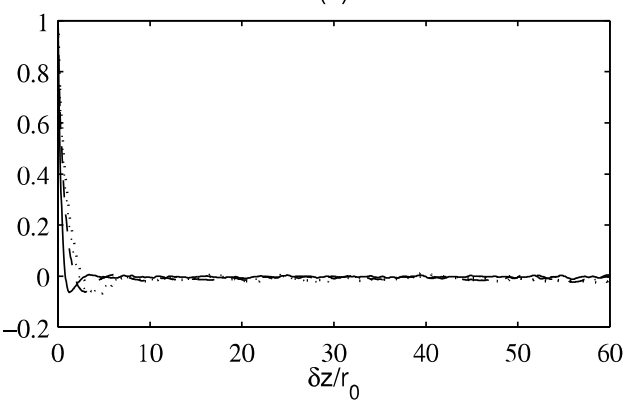

FIgURE 33. Space correlations of axial velocity fluctuations obtained at $(\mathrm{a}, \mathrm{c}) r=0$ and (b,d) $r=r_{0}$ for (top) $\operatorname{Re}_{D}=3,125$ and (bottom) $\operatorname{Re}_{D}=12,500$ : at $-t=10 r_{0} / u_{j}$, $--t=20 r_{0} / u_{j},-\cdot-t=30 r_{0} / u_{j}, \cdots \cdot t=40 r_{0} / u_{j}$.

and pressure spectra at $r=10 r_{0}$ calculated from 5 runs and from 10 runs are compared in figure 34 for the two jets at $\operatorname{Re}_{D}=3,125$ and $\operatorname{Re}_{D}=12,500$, in order to check the sensitivity of the results to the number of runs performed for each jet using different initial perturbations. In all cases, the solutions are identical or very similar to each other. Therefore, the statistical results seem to be well converged in the present study.

\section{Appendix D}

In this final appendix, the influence of the threshold value arbitrarily chosen for the conditional averaging in section 3.5 is illustrated. The fields of velocity and pressure fluctuations obtained at $t=t_{c}+12 r_{0} / u_{j}$ using velocity thresholds of $u_{z}=0.60 u_{j}$, $(2 / 3) u_{j}$ and $0.75 u_{j}$ on the jet axis for the event detection at $t=t_{c}$ are shown in figure 35 for the jet at $\operatorname{Re}_{D}=3,125$ and in figure 36 for the one at $\operatorname{Re}_{D}=12,500$. For both jets, the use of different threshold values is found not to change the results qualitatively. However, lowering the threshold results in an increase of the levels, because only the most energetic events are averaged in this case. For $\operatorname{Re}_{D}=12,500$, for example, the extremum values of velocity fluctuations at $r=0$ and of pressure fluctuations at $r=5 r_{0}$ at $t=t_{c}+12 r_{0} / u_{j}$ are equal to $-0.033 u_{j}$ and $25.3 \mathrm{~Pa}$ using a threshold of $u_{z}=0.75 u_{j}$ but to $-0.079 u_{j}$ and $56.6 \mathrm{~Pa}$ using $u_{z}=0.60 u_{j}$. More oscillations also appear, compare for instance figures $35(\mathrm{a})$ and $35(\mathrm{c})$. Given that the numbers of events detected in the two jets are equal to 62 and 68 using a velocity threshold of $u_{z}=0.75 u_{j}, 45$ and 43 using $u_{z}=(2 / 3) u_{j}$, but only 26 and 23 using $u_{z}=0.60 u_{j}$, they are due to a poorer convergence. 
(a)

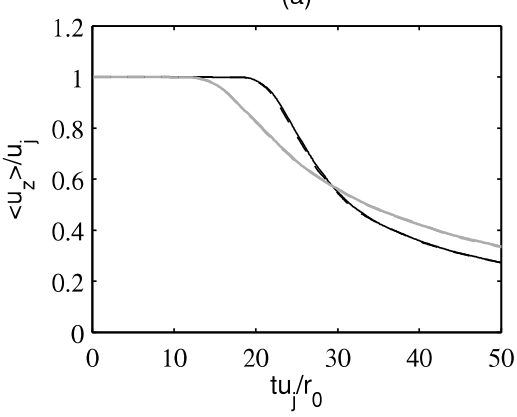

(c)

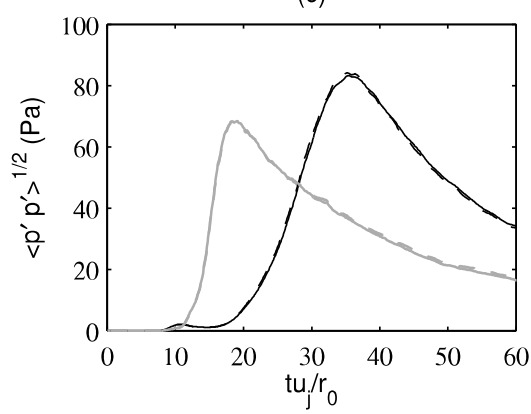

(b)

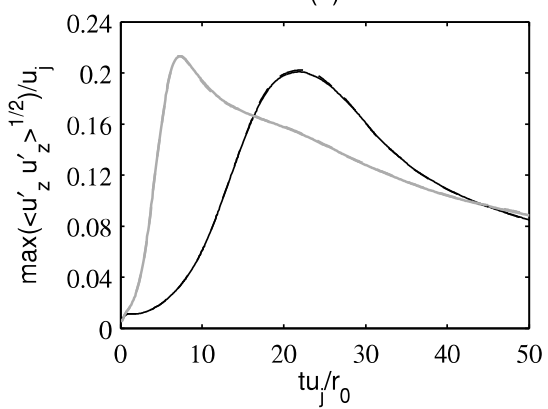

(d)

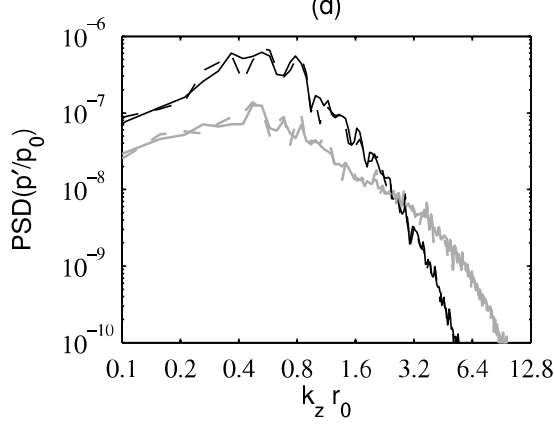

Figure 34. Results obtained for $\operatorname{Re}_{D}=3,125$ from

5 and --10 runs and for $\operatorname{Re}_{D}=12,500$ from $\longrightarrow 5$ and ---10 runs: (a) mean axial velocity at $r=0$, (b) peak value of axial turbulence intensity, (c) rms value of pressure fluctuations at $r=10 r_{0}$, and (d) power spectral densities of pressure fluctuations at $r=10 r_{0}$ at $t u_{j} / r_{0}=45$ for $\operatorname{Re}_{D}=3,125$ and $t u_{j} / r_{0}=40$ for $\operatorname{Re}_{D}=12,500$.

(a)

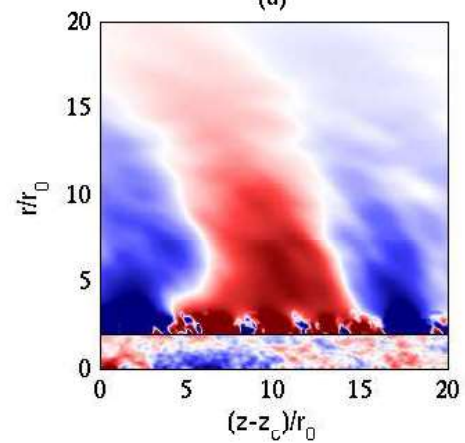

(b)

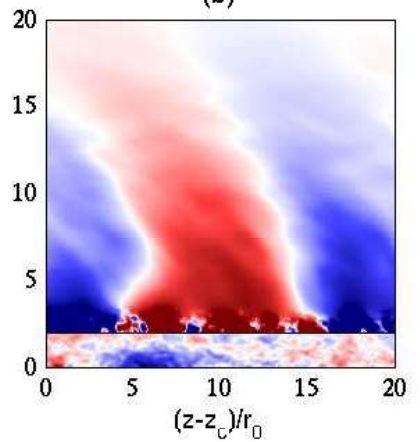

(c)

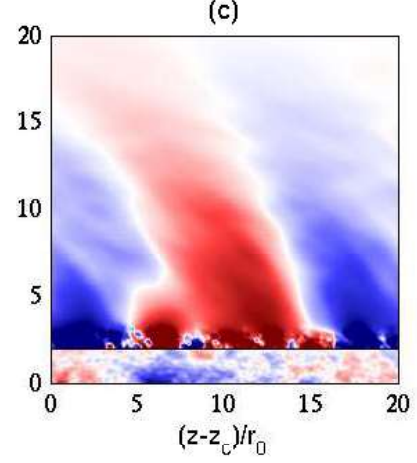

Figure 35. (Colour available at journals.cambridge.org/flm) Velocity and pressure fluctuations obtained at $t=t_{c}+12 r_{0} / u_{j}$ for $\operatorname{Re}_{D}=3,125$ by conditional averaging using thresholds of (a) $u_{z}=0.60 u_{j}$, (b) $u_{z}=(2 / 3) u_{j}$ and (c) $u_{z}=0.75 u_{j}$ for the event detection. The color scales range from $-0.075 u_{j}$ up to $0.075 u_{j}$ and from $-90 \mathrm{~Pa}$ up to $90 \mathrm{~Pa}$, from blue to red.

\section{REFERENCES}

Akamine, M., Okamoto, K., Teramoto, S., Okunuki, T. \& Tsutsumi, S. 2016 Conditional sampling analysis of acoustic phenomena from a supersonic jet impinging on a inclined flat plate. Trans. Japan Soc. Aero. Space Sci. 59 (5), 287-294.

Arndt, R. E. A, Long, D. F. \& Glauser, M. N. 1997 The proper orthogonal decomposition of pressure fluctuations surrounding a turbulent jet. J. Fluid Mech. 340, 1-33. 
(a)

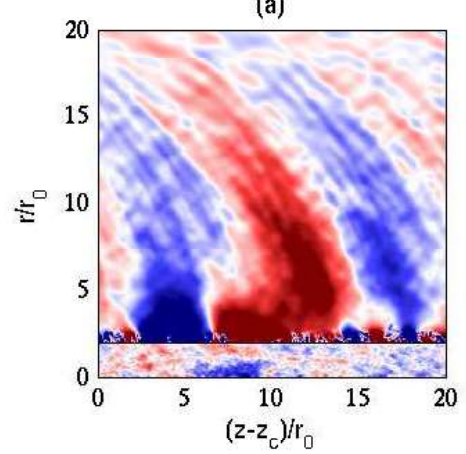

(b)

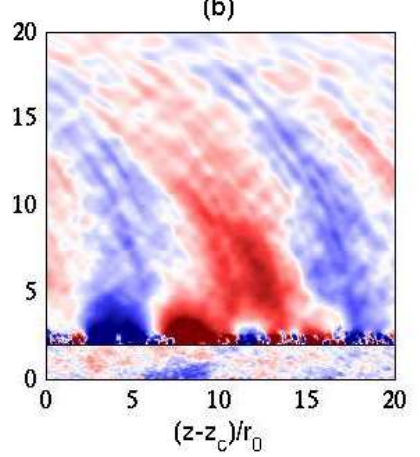

(c)

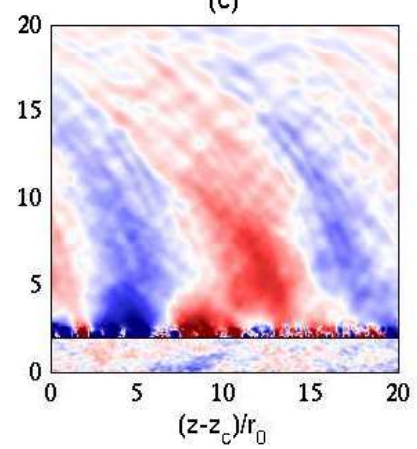

Figure 36. (Colour available at journals . cambridge.org/flm) Velocity and pressure fluctuations obtained at $t=t_{c}+12 r_{0} / u_{j}$ for $\operatorname{Re}_{D}=12,500$ by conditional averaging using thresholds of (a) $u_{z}=0.60 u_{j}$, (b) $u_{z}=(2 / 3) u_{j}$ and (c) $u_{z}=0.75 u_{j}$ for the event detection. The color scales range from $-0.075 u_{j}$ up to $0.075 u_{j}$ and from $-40 \mathrm{~Pa}$ up to $40 \mathrm{~Pa}$, from blue to red.

Berland, J., Bogey, C., Marsden, O. \& Bailly, C. 2007 High-order, low dispersive and low dissipative explicit schemes for multi-scale and boundary problems. J. Comput. Phys. 224 (2), 637-662.

Bogey, C. 2018 Grid sensitivity of flow field and noise of high-reynolds-number jets computed by large-eddy simulation. Int. J. Aeroacoust. p. to appear.

Bogey, C. \& BAILly, C. 2002 Three-dimensional non reflective boundary conditions for acoustic simulations: far-field formulation and validation test cases. Acta Acustica 88 (4), 463471.

Bogey, C. \& Bailly, C. 2004 A family of low dispersive and low dissipative explicit schemes for flow and noise computations. J. Comput. Phys. 194 (1), 194-214.

Bogey, C. \& BAilly, C. 2006 Investigation of downstream and sideline subsonic jet noise using large eddy simulations. Theor. Comput. Fluid Dyn. 20 (1), 23-40.

Bogey, C. \& BAilly, C. 2007 An analysis of the correlations between the turbulent flow and the sound pressure field of subsonic jets. J. Fluid Mech. 583, 71-97.

Bogey, C. \& Bailly, C. 2010 Influence of nozzle-exit boundary-layer conditions on the flow and acoustic fields of initially laminar jets. J. Fluid Mech. 663, 507-539.

Bogey, C., Bailly, C. \& Juvé, D. 2003 Noise investigation of a high subsonic, moderate reynolds number jet using a compressible les. Theor. Comput. Fluid Dyn. 16 (4), 273-297.

Bogey, C., Barré, S., Fleury, V., Bailly, C. \& Juvé, D. 2007 Experimental study of the spectral properties of near-field and far-field jet noise. Int. J. Aeroacoust. 6 (2), 73-92.

Bogey, C., DE CACqueray, N. \& BAilly, C. $2011 a$ Finite differences for coarse azimuthal discretization and for reduction of effective resolution near origin of cylindrical flow equations. J. Comput. Phys. 230 (4), 1134-1146.

Bogey, C. \& Gojon, R. 2017 Feedback loop and upwind-propagating waves in ideallyexpanded supersonic impinging round jets. J. Fluid Mech. 823, 562-591.

Bogey, C. \& MARsden, O. 2016 Simulations of initially highly disturbed jets with experimentlike exit boundary layers. AIAA J. 54 (1), 1299-1312.

Bogey, C., Marsden, O. \& Bailly, C. $2011 b$ Large-eddy simulation of the flow and acoustic fields of a reynolds number $10^{5}$ subsonic jet with tripped exit boundary layers. Phys. Fluids 23 (3), 035104.

Bogey, C., Marsden, O. \& BAilly, C. 2012 Influence of initial turbulence level on the flow and sound fields of a subsonic jet at a diameter-based reynolds number of $10^{5}$. J. Fluid Mech. 701, 352-385.

Buchta, D. A. \& Freund, J. B. 2017 The near-field pressure radiated by planar high-speed free-shear-flow turbulence. J. Fluid Mech. 832, 383-408.

CAmussi, R. \& GRIzzi, S. 2014 Statistical analysis of the pressure field in the near region of a $\mathrm{m}=0.5$ circular jet. Int. J. Aeroacoust. 13 (1), 169-182. 
Cavalieri, A. V. G., Jordan, P., Agarwal, A. \& Gervais, Y. 2011 Jittering wave-packet models for subsonic jet noise. J. Sound Vib. 330 (18-19), 4474-4492.

Chvalieri, A. V. G., Jordan, P., Colonius, T. \& Gervais, Y. 2012 Axisymmetric superdirectivity in subsonic jets. J. Fluid Mech. 704, 388-420.

Chu, W. T. \& Kaplan, R. E. 1976 Use of a spherical concave reflector for jet-noise-source distribution diagnosis. J. Acoust. Soc. Am. 59 (6), 1268-1277.

Coiffet, F., Jordan, P., Delville, J., Gervais, Y. \& Ricaud, F. 2005 Coherent structures in subsonic jets: a quasi-irrotational source mechanism? Int. J. Aeroacoust. 5 (1), 67-89.

Comte, P., Lesieur, M. \& Lamballais, E. 1992 Large- and small-scale stirring of vorticity and a passive scalar in a 3-d temporal mixing layer. Phys. Fluids A 4 (12), 2761-2778.

Crighton, D.G. 1975 Basic principles of aerodynamic noise generation. Prog. Aerospace Sci. $16(1), 31-96$.

Crighton, D. G. 1981 Acoustics as a branch of fluid mechanics. J. Fluid Mech. 106, 261-298.

Crighton, D. G. \& Huerre, P. 1990 Shear layer pressure fluctuations and superdirective acoustic sources. J. Fluid Mech. 220, 355-368.

Dahan, C., Elias, G., Maulard, J. \& Perulli, M. 1978 Coherent structures in the mixing zone of a subsonic hot free jet. J. Sound Vib. 59 (3), 313-333.

Fisher, M. J., Harper-Bourne, M. \& GlegG, S. A. L. 1977 Jet engine noise source location: The polar correlation technique. J. Sound Vib. 51 (1), 23-54.

Fortuné, V., Lamballais, E. \& Gervais, Y. 2004 Noise radiated by a non-isothermal, temporal mixing layer. part i: Direct computation and prediction using compressible dns. Theor. Comput. Fluid Dyn. 18 (1), 61-81.

Freund, J. B. 2001 Noise sources in a low-reynolds-number turbulent jet at mach 0.9. J. Fluid Mech. 438, 277-305.

Freund, J. B., Lele, S. K. \& Moin, P. $2000 a$ Compressibility effects in a turbulent annular mixing layer. part 1. turbulence and growth rate. J. Fluid Mech. 421, 229-267.

Freund, J. B., Lele, S. K. \& Moin, P. $2000 b$ Compressibility effects in a turbulent annular mixing layer. part 2. mixing of a passive scalar. J. Fluid Mech. 421, 269-292.

Grizzi, S. \& CAmussi, R. 2012 Wavelet analysis of near-field pressure fluctuations generated by a subsonic jet. J. Fluid Mech. 698, 93-124.

Hileman, J., Thurow, B., Caraballo, E. J. \& Samimy, M. 2005 Large-scale structure evolution and sound emission in high-speed jets: Real-time visualization with simultaneous acoustic measurements. J. Fluid Mech. 544, 277-307.

Jordan, P. \& Colonius, T. 2013 Wave packets and turbulent jet noise. Annu. Rev. Fluid Mech. 45, 173-195.

Juvé, D., Sunyach, M. \& Comte-Bellot, G. 1979 Filtered azimuthal correlations in the acoustic far field of a subsonic jet. AIAA J. 17 (1), 112-113.

Juvé, D., Sunyach, M. \& Comte-Bellot, G. 1980 Intermittency of the noise emission in subsonic cold jets. J. Sound Vib. 71 (3), 319-332.

Kastner, J., Samimy, M., Hileman, J. \& Freund, J. B. 2006 Comparison of noise mechanisms in high and low reynolds number high-speed jets. AIAA J. 44 (10), 2251-2258.

Kearney-Fisher, M., Sinha, A. \& Samimy, M. 2013 Intermittent nature of subsonic jet noise. AIAA J. 51 (5), 1142-1155.

Keiderling, F., Kleiser, L. \& Bogey, C. 2009 Numerical study of eigenmode forcing effects on jet flow development and noise generation mechanisms. Phys. Fluids 21 (4), 045106

Kempton, A. J. \& Ffowcs Williams, J. E. 1978 The noise from the large-scale structure of a jet. J. Fluid Mech. 84 (4), 673-694.

Kleimann, R. R. \& Freund, J. B. 2008 The sound from mixing layers simulated with different ranges of turbulence scales. Phys. Fluids 20 (10), 101503.

Lighthill, M. J. 1952 On sound generated aerodynamically i. general theory. Proc. Roy. Soc. A 211 (1107), 564-587.

MichalKe, A. 1964 On the inviscid instability of the hyperbolic-tangent velocity profile. $J$. Fluid Mech. 19 (4), 543-556.

Michalke, A. 1984 Survey on jet instability theory. Prog. Aerospace Sci. 21, 159-199.

Mohseni, K. \& Colonius, T. 2000 Numerical treatment of polar coordinate singularities. J. Comput. Phys. 157 (2), 787-795. 
Mollo-Christensen, E. 1967 Jet noise and shear flow instability seen from an experimenter's viewpoint. J. Appl. Mech 34 (1), 1-7.

Mollo-Christensen, E., Kolpin, M. A. \& Martucelli, J. R. 1964 Experiments on jet flows and jet noise far-field spectra and directivity patterns. J. Fluid Mech. 18, 285-301.

Morris, P. J. 1976 The spatial viscous instability of axisymmetric jets. J. Fluid Mech. 77 (3), $511-529$.

PANDA, J. 2007 Experimental investigation of turbulent density fluctuations and noise generation from heated jets. J. Fluid Mech. 591, 73-96.

Panda, J., Seasholtz, R. G. \& Elam, K. A. 2005 Investigation of noise sources in high-speed jets via correlation measurements. J. Fluid Mech. 537, 349-385.

Papamoschou, D. 2018 Wavepacket modeling of the jet noise source. Int. J. Aeroacoust. 17 (12), 52-69.

Powell, A. 1964 Theory of vortex sound. J. Acoust. Soc. Am. 36 (1), 177-195.

RAGAB, S. A. \& WU, J. L. 1989 Linear instabilities in two-dimensional compressible mixing layers. Phys. Fluids A 1 (6), 957-966.

van ReeuniJk, M. \& Holzner, M. 2014 The turbulence boundary of a temporal jet. J. Fluid Mech. 739, 254-275.

Ribner, H. S. 1964 The generation of sound by turbulent jets. Adv. Appl. Mech. 8, 103-182.

SCHAFFAR, M. 1979 Direct measurements of the correlation between axial in-jet velocity fluctuations and far field noise near the axis of a cold jet. J. Sound Vib. 64 (1), 73-83.

SeIner, J. M. 1974 The distribution of jet source strength intensity by means of direct correlation technique. PhD thesis, Pennsylvania State University.

Stromberg, J. L., Mclaughlin, D. K. \& Troutt, T. R. 1980 Flow field and acoustic properties of a mach number 0.9 jet at a low reynolds number. J. Sound. Vib. 72 (2), $159-176$

Suzuki, T. 2010 Review of diagnostic studies on jet-noise sources and generation mechanisms of subsonically-convecting jets. Fluid Dyn. Res. 42, 014001.

SuZuki, T. 2013 Coherent noise sources of a subsonic round jet investigated using hydrodynamic and acoustic phased-microphone arrays. J. Fluid Mech. 730, 659-698.

TAM, C. K. W. 1995 Supersonic jet noise. Annu. Rev. Fluid Mech. 27, 17-43.

TAM, C. K. W. 1998 Jet noise: Since 1952. Theor. Comput. Fluid Dyn. 10 (1-4), 393-405.

TAm, C. K. W. \& Auriault, L. 1999 Jet mixing noise from fine-scale turbulence. AIAA J. $37(2), 145-153$.

TAM, C. K. W \& Dong, Z. 1996 Radiation and outflow boundary conditions for direct computation of acoustic and flow disturbances in a nonuniform mean flow. J. Comput. Acoust. 4 (2), 175-201.

Tam, C. K. W., Golebiowski, M. \& Seiner, J. M. 1996 On the two components of turbulent mixing noise from supersonic jets. AIAA Paper 96-1716.

TAM, C. K. W. \& MorRIS, P.J. 1980 The radiation of sound by instability waves of a compressible plane turbulent shear layer. J. Fluid Mech. 98 (2), 349-381.

Tam, C. K. W., Viswanathan, K., Ahuja, K. K. \& Panda, J. 2008 The sources of jet noise: experimental evidence. J. Fluid Mech. 615, 253-292.

Tinney, C. E., Ukeiley, L. S. \& Glauser, M. N. 2008 Low-dimensional characteristics of a transonic jet. part 2. estimate and far-field prediction. J. Fluid Mech. 615, 53-92.

Ukeiley, L. \& Ponton, M. K. 2004 On the near field pressure of a transonic axisymmetric jet. Int. J. Aeroacoust. 3 (1), 43-65.

Vreman, A. W., Sandham, N. D. \& Luo, K. H. 1996 Compressible mixing layer growth rate and turbulence characteristics. J. Fluid Mech. 320, 235-258.

Winant, C. D. \& Browand, R. K. 1974 Vortex pairing : the mechanism of turbulent mixinglayer growth at moderate reynolds number. J. Fluid Mech. 63 (2), 237-255.

Zaman, K. B. M. Q. 1985 Effect of initial condition on subsonic jet noise. AIAA J. 23 (9), 1370-1373.

Zaman, K. B. M. Q. 1986 Flow field and near and far sound field of a subsonic jet. J. Sound Vib. 106 (1), 1-16. 